\title{
Transcriptional Effects of E3 Ligase Atrogin-1/MAFbx on Apoptosis, Hypertrophy and Inflammation in Neonatal Rat Cardiomyocytes
}

\author{
Yong Zeng ${ }^{1 *}$, Hong-Xia Wang ${ }^{2}$, Shu-Bin Guo ${ }^{3}$, Hui Yang ${ }^{2}$, Xiang-Jun Zeng ${ }^{2}$, Quan Fang ${ }^{1}$, Chao- \\ Shu Tang ${ }^{2}$, Jie $\mathrm{Du}^{4}$, Hui-Hua $\mathrm{Li}^{2 *}$
}

1 Department of Cardiology, Peking Union Medical Hospital, Beijing, China, 2 The Key Laboratory of Remodeling-related Cardiovascular Diseases, Department of Pathology and Pathophysiology, School of Basic Medical Sciences, Capital Medical University, Beijing, China, 3 Department of Emergency Medicine, Peking Union Medical Hospital, Beijing, China, 4 The Key Laboratory of Remodeling-related Cardiovascular Diseases, Laboratory of Vascular Biology, Beijing Institute of Heart Lung and Blood Vessel Diseases, Beijing Anzhen Hospital Affiliated the Capital Medical University, Beijing, China

\begin{abstract}
Atrogin-1/MAFbx is an ubiquitin E3 ligase that regulates myocardial structure and function through the ubiquitin-dependent protein modification. However, little is known about the effect of atrogin-1 activation on the gene expression changes in cardiomyocytes. Neonatal rat cardiomyocytes were infected with adenovirus atrogin-1 (Ad-atrogin-1) or GFP control (AdGFP) for 24 hours. The gene expression profiles were compared with microarray analysis. 314 genes were identified as differentially expressed by overexpression of atrogin-1, of which 222 were up-regulated and 92 were down-regulated. Atrogin-1 overexpression significantly modulated the expression of genes in 30 main functional categories, most genes clustered around the regulation of cell death, proliferation, inflammation, metabolism and cardiomyoctye structure and function. Moreover, overexpression of atrogin-1 significantly inhibited cardiomyocyte survival, hypertrophy and inflammation under basal condition or in response to lipopolysaccharide (LPS). In contrast, knockdown of atrogin-1 by siRNA had opposite effects. The mechanisms underlying these effects were associated with inhibition of MAPK (ERK1/2, JNK1/2 and p38) and NF-KB signaling pathways. In conclusion, the present microarray analysis reveals previously unappreciated atrogin-1 regulation of genes that could contribute to the effects of atrogin-1 on cardiomyocyte survival, hypertrophy and inflammation in response to endotoxin, and may provide novel insight into how atrogin-1 modulates the programming of cardiac muscle gene expression.
\end{abstract}

Citation: Zeng Y, Wang H-X, Guo S-B, Yang H, Zeng X-J, et al. (2013) Transcriptional Effects of E3 Ligase Atrogin-1/MAFbx on Apoptosis, Hypertrophy and Inflammation in Neonatal Rat Cardiomyocytes. PLoS ONE 8(1): e53831. doi:10.1371/journal.pone.0053831

Editor: Tianqing Peng, University of Western Ontario, Canada

Received October 5, 2012; Accepted December 3, 2012; Published January 15, 2013

Copyright: (c) 2013 Zeng et al. This is an open-access article distributed under the terms of the Creative Commons Attribution License, which permits unrestricted use, distribution, and reproduction in any medium, provided the original author and source are credited.

Funding: This study was supported by grants from the China Natural Science Foundation (81025001, 30971097 and 30888004$)$, the Beijing High-Level Talents Program (PHR20110507). The funders had no role in study design, data collection and analysis, decision to publish, or preparation of the manuscript.

Competing Interests: The authors have declared that no competing interests exist.

*E-mail: zengyong1999@yahoo.com (YZ); hhli1935@yahoo.cn (HHL)

\section{Introduction}

Heart failure (HF) is the final and common pathway for various cardiovascular diseases. Lipopolysaccharide (LPS) from gram negative bacteria is one of the most common causes of inflammation and innate immunity through Toll-like receptor-4 (TLR-4) [1,2]. Several studies demonstrated that LPS directly induces cardiomyocyte hypertrophy, apoptosis and depresses contractility, ultimately leading to cardiomyopathy and congestive heart failure [3]. Accumulating evidence has shown that cardiac muscle mass loss, because of increased rate of protein degradation and cellular death, represents a critical pathogenic event in $\mathrm{HF}$ [4]. Disturbances in the ubiquitin-proteasome system are thought to be involved in the development of various cardiovascular diseases, including HF, cardiac infarction and atherosclerosis [48]. Recently, a study has identified two novel ubiquitin E3 ligases, atrogin-1/MAFbx and MuRF1, which function as negative regulator of muscle cell size in vitro and in vivo [5,6,9-12].

Atrogin-1 is an F-box protein selectively expressed in cardiac and skeletal muscle tissues, and is known to be up-regulated markedly in skeletal muscle in a variety of models of catabolic states, including oxidative stress, fasting, cancer, sepsis, heart failure [810,13,14]. Importantly, atrogin-1 associates with Skp1, Cull and Rocl to form an SKP1-CUL1-F-box (SCF)-type ubiquitin ligase $[5,6,10,15]$. Overexpression of atrogin- 1 in skeletal myotubes leads to atrophy, whereas atrogin-1 deficiency results in marked resistance to skeletal muscle denervation atrophy [10]. Moreover, atrogin-1 confers SCF complex specificity by directly targeting many substrates including calcineurin, initiation factor eIF3-f, Myo D, and mitogen-activated protein kinase phosphatase-1 (MKP-1) for proteasome-dependent degradation, leading to negative regulation of muscle cell size and cardiomyocte survival [5,16-19]. However, We have recently described a novel role for atrogin- 1 as a co-activator of FOXO1/3a through lysine 63-linked polyubiquitination, thereby inhibiting Akt-dependent physiologic hypertrophy [6]. These results suggest that atrogin-1 plays a pivotal role in muscle atrophy, cardiac hypertrophy and cardiomyocyte apoptosis.

Although it is well known that upstream proteins including Foxol/3a that activate atrogin-1 transcription and enhance its 
activity for protein degradation are required for heart failure $[15,20,21]$, little is known about how atrogin-1 contributes cardiomyocyte apoptosis, proliferation and hypertrophy through regulating gene expression at the transcriptional level. To gain insight into the early molecular events associated with atrogin-1mediated cardiomyocyte apoptosis, hypertrophy and inflammation, we used primary neonatal rat cardiomyocytes as a model to investigate the effects of atrogin-1 on cardiac muscle gene expression, and confirmed the results by real-time quantitative PCR analysis. Our results showed that atrogin-1 overexpression modulated the expression of many genes involved in the regulation of diverse biological functions, including cell survival, proliferation, inflammation, cell metabolism and cardiac hypertrophy. Importantly, atrogin-1 inhibits endotoxin LPS-induced cardiomyocyte apoptosis, hypertrophy and inflammation through mitogen activated protein kinases (MAPKs) and NF- $\mathrm{KB}$ signaling pathways. We believe that such a comprehensive analysis of gene expression profile in neonatal rat cardiomyocytes may identify novel targets for drug discovery in the intervention of the progression of cardiac dysfunction.

\section{Results}

\section{Effects of atrogin-1 overexpression on gene expression profiles in neonatal rat cardiomyocytes}

To identify genes differentially regulated by atrogin-1 in cardiomyocytes, we examined the changes in the gene expression profiles of cardiomyocytes infected by adenovirus atrogin-1 (Adatrogin-1) and GFP control (Ad-GFP) using DNA microarray assay. After 24 hours, the infection efficiency was more than $95 \%$ as characterized by GFP, the level of atrogin-1 protein in cardiomyocytes was increased by 2.8-fold compared to Ad-GFP control (Figure 1A and B). Moreover, we found that overexpression of atrogin-1 in cultured cardiomyocytes leads to 314 genes being significantly regulated when compared to control group. Of them, 222 were up-regulated and 92 down-regulated by atrogin-1 overexpression. A hierachical clustering on the complete set of data of present genes was performed as described [22]. The analysis showed a clear separation of the Ad-GFP control $(G)$ and overexpressed atrogin-1 (A) groups, when a clustering was performed on a subset of genes that displayed different expression with fold changes $\geq 2$-fold or $\leq-2$-fold (Figure $1 \mathrm{C}$ ).

\section{Validation of microarray analysis with qRT-PCR}

To examine the reliability of microarray results, we performed qRT-PGR analysis for a set of eight genes including Axin2, Cxcl6, Calr, IL-1r1, Cadm1, Cxcl1, Dkk2 and IL-6, which were differentially expressed in the microarray assay. Scatter plot analysis of the relative changes in expression as determined by qRT-PGR and microarray revealed a good correlation (Figure 2).

To validate the microarray data, we performed qRT-PCR for a set of 10 genes that were differentially expressed in atrogin-1infected cardiomyocytes. 7 of 10 genes showed similar expression ratios between qRT-PCR data and microarray results. Overexpressed atrogin-1 significantly induced the transcription of cell proliferation-related genes including the Axin2, Calr and DKK2, and down-regulated the transcriptional level of cardiac remodeling-related gene MMP3, adhesion-related gene Cadml and chemotaxis-related genes including Cxcl1, Cxcl6 and IL-6 compared to Ad-GFP control (Figure 3A). In contrast, knockdown of atrogin- 1 by siRNA significantly decreased the transcriptional level of Calr, Axin2 and Ccnd2, and up-regulated the transcriptional level of Cadm1, Gcl20, Cxcl1, Cxcl2, Cxcl6 and IL-6 compared to siRNA-control (Figure 3B). Collectively, these results further provide evidence to support the validity and quality of the microarray data.

\section{Functional characterization of the atrogin-1-regulated genes}

Next, we clustered atrogin-1-regulated genes into functional groups using gene annotation information from the Affymetrix database. Our data showed that the most prominently enriched biological process in the differentially atrogin-1-regulated genes was cell apoptosis, proliferation, metabolism, hypertrophy and inflammation.

\section{Genes involved in cell apoptosis}

Twenty-two genes were differentially regulated by atrogin-1 overexpression (Table 1). Eight of them including Csf2 [23-25], IL-6 [26-28] and Aldhla3 [29] were down-regulated, which were involved in negative regulation of apoptosis. Conversely, fourteen of them including Msx2 [30] and Axin2 [31] were up-regulated, which mediated positive regulation of apoptosis.

\section{Genes involved in cell proliferation}

One of the most significant overrepresented terms of biological process was cell proliferation in Ad-atrogin-1 group compared to Ad-GFP control group. Eighteen genes were differentially regulated, which are involved in cell proliferation (Table 2). Of them, thirteen genes such as Ptgs2 [32] were down-regulated, which mediated positive regulation of cell proliferation. In contrast, seven genes such as Axin2 [31], Msx2 [30] and Prkcz $(\mathrm{PKC \varepsilon})$ [33] involved in negative regulation of cell proliferation and cell cycle arrest were up-regulated.

\section{Genes involved in metabolisms}

Metabolic pathways was a crucial signaling pathway through which sixteen genes were significantly enriched (Table 3). Twelve genes such as Ptgis [34] and Pycrl [35] were up-regulated. Conversely, four genes including Ckmtl and Ptges were downregulated, which mediates negative regulation of mitochondrial function [36].

\section{Genes involved in cell development and hypertrophic cardiomyopathy}

Seventeen genes were closely related to the regulation of cell development (Table 4). Thirteen of them such as Myh6 ( $\alpha$-MHC) [37], Bhlha15 (Mist1) [38] and Serca2 (ATP2A2) [39,40] and Prkcz that are involved in cell maturation, sarcomere organization and hypertrophy were up-regulated. Four of them such as Foxa2 [41] and ITGA3 were down-regulated, which mediate regulation of gene expression in response to insulin stimulus and cell differentiation.

\section{Genes involved in inflammation}

Response to inflammation and stress was a prominently overrepresented GO term in which thirty-five genes were involved (Table 5), nineteen of them involved in inflammatory response were significantly down-regulated by overexpression of atrogin-1, these genes include IL-6 [42], Ptger3 [43], Lbp [44], Ptgs2 (Cox-2) [45] and chemokines such as Cxcl1, Cxcl2, Cxcl3, Ccl20 and Cxcl6 [46,47]. However, sixteen of them such as IL-1rl was significantly up-regulated [48]. 
A

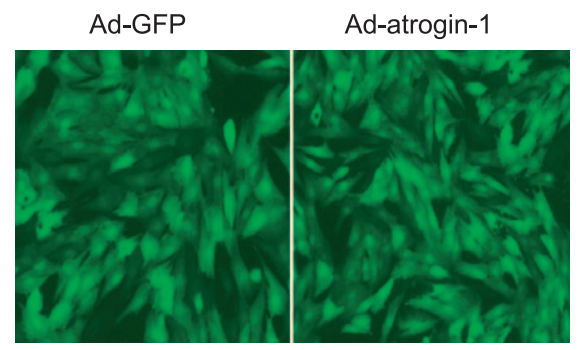

B

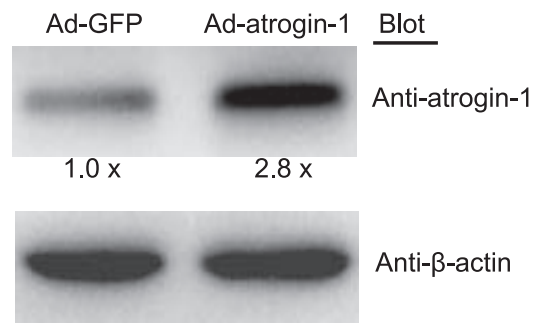

C

\section{Hierarchical clustering}
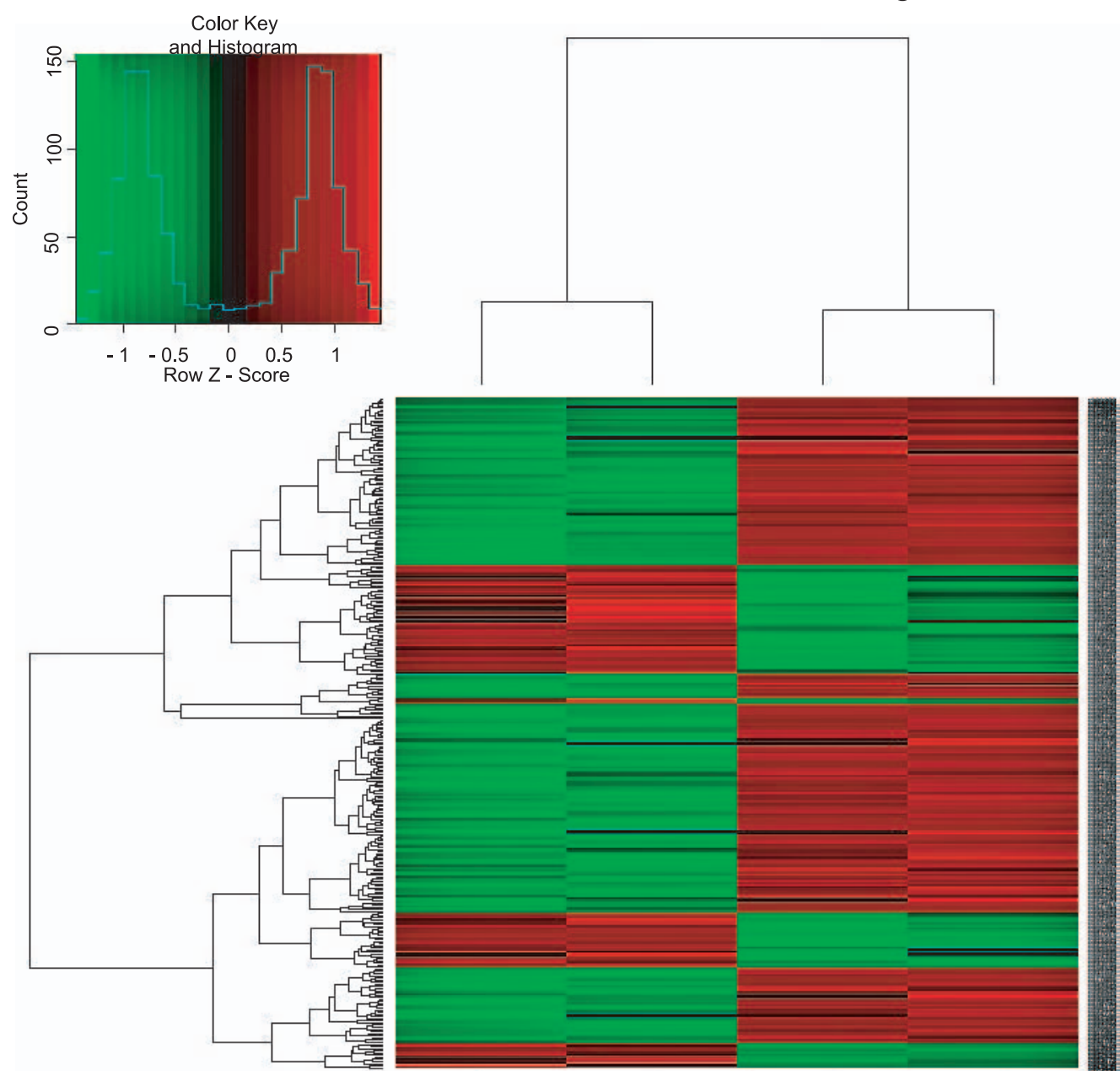

G1

G2

A2

A1

Figure 1. Infection of adenovirus atrogin-1 and microarray analysis. A. Neonatal rat cardiomyocytes were infected with adenovirus green fluorescent protein (GFP) control (Ad-GFP) or atrogin-1 (Ad-atrogin-1). The infection efficiency was visualized for GFP 24 hours later using fluorescence microscopy (Magnification, $\times 400$ ). B. The levels of atrogin-1 protein were determined by Western blot analysis with anti-atrogin-1 antibody, using $\beta$ actin as the internal control. Quantitative analysis of protein bands was shown $(n=3)$. C. Hierarchical clustering depicting expression profiles of 
differentially expressed genes in Ad-atrogin-1 (A1 and A2) and Ad-control (G1 and G2) groups. Data from individual sample are shown. A subset of genes displays significant expression changes at $\geq 2$-fold or $\leq-2$-fold. Gene expression levels are shown as color variations (red: up-regulated expression; green: down-regulated expression).

doi:10.1371/journal.pone.0053831.g001

\section{Effects of atrogin-1 on cardiomyocyte apoptosis, hypertrophy and inflammation}

Since genes differentially regulated by atrogin- 1 in cardiomyocytes have been implicated in cell apoptosis, hypertrophy and inflammation (Table 1, 4 and 5), which cause cardiac dysfunction and ultimately lead to heart failure, we then investigated whether overexpression of atrogin-1 influences cardiomyocyte apoptosis. Consistent with previous reports [18], atrogin-1 overexpression significantly increased TUNEL-positive cardiomyocytes compared to Ad-GFP control (Figure 4A). After LPS stimulation, TUNELpositive cardiomyocytes were significantly increased, and this effect was further enhanced by Ad-atrogin-1 infection (Figure 4A). Moreover, consistent with our previous reports [5,6,49], atrogin-1 overexpression markedly reduced cardiomyocyte surface areas under basal condition and in response to LPS stimulation (Figure 4B). qPCR analysis of hypertrophic markers (ANF, aMHC and Serca2) further confirmed the effect of atrogin-1 on cardiomyocyte hypertrophy (Figure 4C). In contrast, knockdow of atrogin- 1 by siRNA signaficantly reversed these effcts under basal condition or in response to LPS (Figure 4D and E). Finally, atrogin1 overexpression markedly inhibited the expression of proinflammatory-related genes (including IL-1 $\beta$, IL-6, Ptgs2 and Serpinb2) and chemokines (Cxcl1, Cxcl2 and Ccl20) but upregulated anti-inflammatory gene IL-1r1 expression compared to Ad-GFP control under basal condition or in response to LPS stimulation (Figure 5A), whereas these effects were reversed by knockdown of atrogin-1 (Figure 5B). These results further confirmed the data from microarray analysis (Table 5). Collectively, these results indicate that increased expression of atrogin-1 inhibits cardiomyocyte survival, hypertrophy and inflammation under basal condition or in response to LPS stimulation.

\section{Effects of atrogin-1 on MAPKs and NF- $\mathrm{KB}$ signaling pathways}

To elucidate the mechanisms for atrogin- 1 to regulate transcriptional gene expression, we examined the activation of two major signaling pathways of MAPK and NF- $\kappa \mathrm{B}$, which play critical roles in controlling the expression of apoptosis-, hypertrophy- and inflammation-related genes [50]. Our results showed that under saline treatment, overexpression of atrogin- 1 decreased the levels of ERK1/2, JNK1/2, p38 and p-65 phosphorylation protein compared to Ad-GFP control (Figure 6A). After LPS stimulation, the levels of ERK1/2, JNK1/2, p38 and p-65 phosphorylation were significantly increased compared to Ad-GFP control, However, LPS-induced effects were markedly attenuated by overexpression of atrogin-1 in cardiomyocytes (Figure 5A). In contrast, depletion of atrogin-1 by siRNA had opposite effects (Figure 6B). Together, these results suggest that MAPKs and NF$\kappa \mathrm{B}$ signaling pathways could be attributed to the effects of atrogin-1 in cardiomyocytes under basal condition or after LPS treatment.

\section{Discussion}

The present results demonstrate that atrogin- 1 activation results in the differential regulation of 314 genes in neonatal rat cardiomyocytes, of which 222 were up-regulated and 92 were down-regulated. Interestingly, the majority of differentially and highly expressed genes were involved in cell death, proliferation, inflammation, metabolism and cardiomyopathy. Moreover, in-

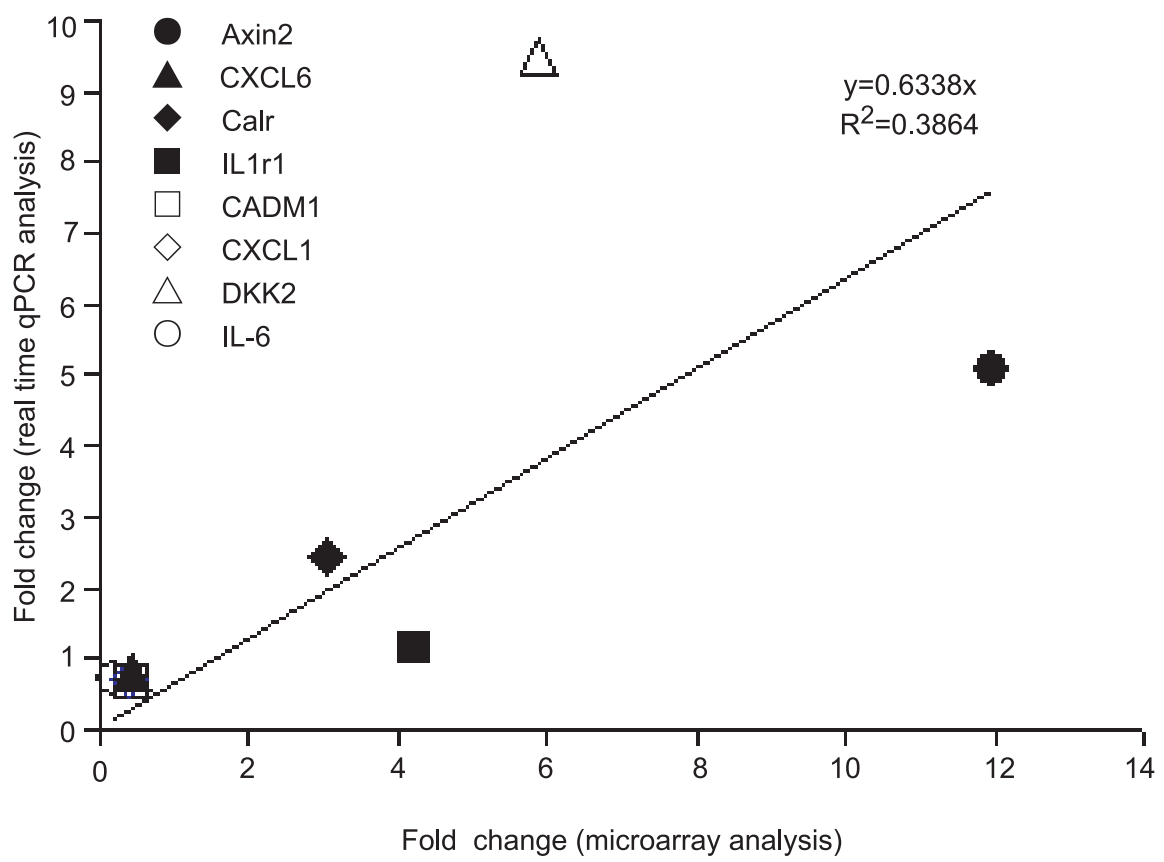

Figure 2. Scatter plot of relative changes in gene expression as determined by microarray analysis and by qRT-PCR. Eight genes were analyzed: IL-6, DKK2, Calr, Cxcl6, Cxcl1, Axin2, IL-1r1, and Cadm1. Each symbol represents the fold change of the respective gene in ad-atrogin-1 group over Ad-GFP control.

doi:10.1371/journal.pone.0053831.g002 

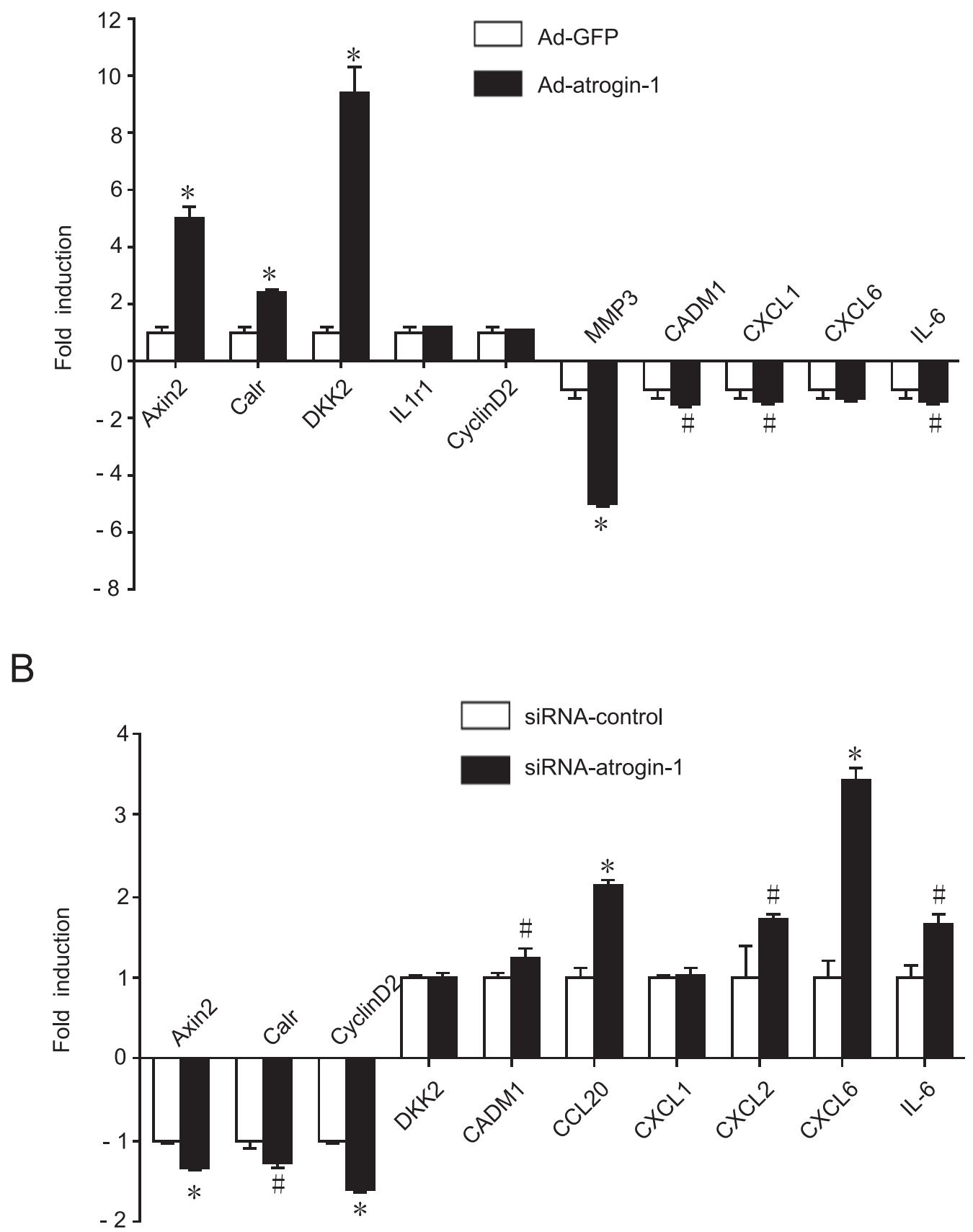

Figure 3. qRT-PCR analysis of microarray data. Neonatal rat cardiomyocytes were infected with Ad-atrogin-1 or Ad-GFP (A), Ad-siRNA-atrogin-1 or Ad-siRNA-control (B) for 24 hours. Total RNA was extracted and qRT-PCR analysis was performed in triplicate using specific oligonucleotides primers. The differences in gene expression levels were statistically significant. Data represent the mean $\pm \operatorname{SEM}\left(n=3\right.$ per group). ${ }^{\#} P<0.05 ;{ }^{*} P<0.001$ vs. Ad-GFP control.

doi:10.1371/journal.pone.0053831.g003

creased expression of atrogin-1 significantly inhibited caerdiomyocyte survival, hypertrophy and inflammation under basal condition or in response to LPS stimulation. The mechanisms underlying these effects were associated with inhibition of MAPK (ERK1/2, JNK1/2 and p38) and NF- $\mathrm{BB}$ signaling pathways.

Emerging evidences suggest that atrogin-1 is a muscle-specific E3 ligase and is up-regulated by various of catabolic conditions [810,12,51]. Recent studies demonstrate that TNF- $\alpha$, doxorubicin
(DOX) and LPS can stimulate atrogin-1 mRNA expression and ubiquitin conjugating activity in skeletal muscle through activation of p38 MAPK [51-53]. In contrast, AKT activation decreases atrogin-1 expression through inhibition of FoxO transcriptional factors [54]. In addition, atrogin-1 protein is degradated by proteasome via p38 MAPK signaling pathway [55]. These data indicate that multiple signaling pathways regulate atrogin-1 expression at different levels. 
Table 1. GO:0008219 cell apoptosis and death.

\begin{tabular}{|c|c|c|c|}
\hline Gene Symbol & Gene Title & Fold change & $P$ value \\
\hline Rab27a & RAB27A, member RAS oncogene family & 5.69 & 0.0011 \\
\hline Prkcz & protein kinase $C$, zeta & 3.66 & $4.0 \mathrm{E}-05$ \\
\hline Msx2 & msh homeobox 2 & 3.59 & 0.027 \\
\hline Herpud1 & homocysteine-inducible, endoplasmic reticulum stress-inducible, ubiquitin -like $d$ & 3.47 & 5.7E-05 \\
\hline Dnajb9 & DnaJ (Hsp40) homolog, subfamily B, member 9 & 3.27 & $6.1 \mathrm{E}-05$ \\
\hline Hspa5 & heat shock protein 5 & 3.19 & $2.8 \mathrm{E}-04$ \\
\hline Tcf7 & transcription factor 7 , T-cell specific & 2.82 & 0.0017 \\
\hline Pdia3 & protein disulfide isomerase family $A$, member 3 & 2.69 & $1.3 \mathrm{E}-05$ \\
\hline Pdia2 & protein disulfide isomerase famil & 2.33 & 0.0039 \\
\hline Sels & selenoprotein S & 2.29 & $1.5 \mathrm{E}-04$ \\
\hline Eif2ak3 & eukaryotic translation initiation factor 2 alpha kinase 3 & 2.14 & $3.1 \mathrm{E}-04$ \\
\hline Gclc & glutamate-cysteine ligase, catalytic subunit & 2.05 & 0.018 \\
\hline Psen2 & presenilin 2 & -2.02 & $7.8 \mathrm{E}-04$ \\
\hline Aldh1a3 & aldehyde dehydrogenase 1 family, member $A 3$ & -2.04 & 0.05 \\
\hline Mapt & microtubule-associated protein tau & -2.17 & 0.027 \\
\hline Cadm1 & cell adhesion molecule 1 & -2.34 & 0.0026 \\
\hline Ptgs2 & prostaglandin-endoperoxide synthase 2 & -3.56 & 0.020 \\
\hline Csf2 & colony stimulating factor 2 (granulocyte-macrophage) & -3.78 & 0.042 \\
\hline II-6 & interleukin 6 & -4.83 & 0.006 \\
\hline
\end{tabular}

doi:10.1371/journal.pone.0053831.t001

Apoptotic cell death in cardiac myocytes appears to be an early event and is well recognized to be responsible for several cardiovascular diseases such as $\mathrm{HF}$ and myocardial infarction [56]. LPS, one of the most common causes of inflammation, directly induces cardiomyocyte apoptosis and hypertrophy through calcineurin signaling pathway [3,49,51]. However, it is unclear that whether atrogin-1 contributes to LPS-induced cardiomyocyte apoptosis. Recently, our data show that atrogin-1 plays an important role in regulating cardiomyocyte apoptosis.
Overexpression of atrogin-1 promotes ischemia/reperfusion (I/R)induced cardiomyocyte apoptosis through activation of JNK signaling pathway [18]. However, the mechanisms by which atrogin- 1 promotes cardiomyocyte apoptosis remain to be elucidated. In this study, we examined the gene expression profiling regulated by atrogin-1 overexpression in cardiomyocytes. Our microarray data showed that many genes involved in cell apoptosis and proliferation, including Csf2, IL-6, Ptgs2, Aldh1a3, Axin2, Msx2, Prkcz, Calr, and Pdia3 were differentially regulated (Table 1

Table 2. GO:0008283 cell proliferation.

\begin{tabular}{|c|c|c|c|}
\hline Gene Symbol & Gene Title & Fold change & P value \\
\hline Axin2 & $\operatorname{axin} 2$ & 12.2 & $6.6 \mathrm{E}-06$ \\
\hline Prkcz & protein kinase $C$, zeta & 3.66 & 4.0E-05 \\
\hline Msx2 & msh homeobox 2 & 3.59 & 0.027 \\
\hline Abcc4 & ATP-binding cassette, sub-family C (CFTR/MRP), member 4 & 3.20 & $1.3 \mathrm{E}-04$ \\
\hline Calr & calreticulin & 3.05 & $1.2 \mathrm{E}-04$ \\
\hline Mycn & v-myc myelocytomatosis viral related oncogene, neuroblastoma derived (avian) & 2.85 & $1.5 \mathrm{E}-04$ \\
\hline Tcf7 & transcription factor $7, \mathrm{~T}$-cell specific & 2.82 & 0.0017 \\
\hline Fgf9 & fibroblast growth factor 9 & 2.42 & 0.0038 \\
\hline Ccnd2 & cyclin D2 & 2.21 & 0.0067 \\
\hline Cdk5rap3 & CDK5 regulatory subunit associated protein 3 & 2.03 & 0.0021 \\
\hline Klf5 & Kruppel-like factor 5 & -2.17 & 0.013 \\
\hline Cadm1 & cell adhesion molecule 1 & -2.34 & 0.0026 \\
\hline Ptges & prostaglandin E synthase & -3.21 & 0.05 \\
\hline Ptgs2 & prostaglandin-endoperoxide synthase 2 & -3.56 & 0.020 \\
\hline Csf2 & colony stimulating factor 2 (granulocyte-macrophage) & -3.78 & 0.04 \\
\hline
\end{tabular}


Table 3. Metabolic pathways.

\begin{tabular}{llll}
\hline & & & \\
\hline Gene Symbol & Gene Title & Fold change & P value \\
\hline Isyna1 & inositol-3-phosphate synthase 1 & 4.09 & $3.8 \mathrm{E}-05$ \\
\hline Gmppb & GDP-mannose pyrophosphorylase B & $2.6 \mathrm{E}-06$ \\
Pycr1 & pyrroline-5-carboxylate reductase 1 & 3.62 & 0.004 \\
LOC684425 & similar to Adenylosuccinate synthetase isozyme 1 & 0.05 \\
Alg12 & asparagine-linked glycosylation 12 homolog & 2.57 & $4.4 \mathrm{E}-05$ \\
Nans & N-acetylneuraminic acid synthase & 2.27 & $2.2 \mathrm{E}-04$ \\
Oat & ornithine aminotransferase (gyrate atrophy) & $3.8 \mathrm{E}-04$ \\
Rpn1 & ribophorin I & 2.26 & $7.5 \mathrm{E}-06$ \\
Slc33a1 & Solute carrier family 33 & 2.25 & $5.9 \mathrm{E}-04$ \\
Stt3a & STT3, subunit of the oligosaccharyltransferase complex, homolog A & 0.002 \\
Gclc & glutamate-cysteine ligase, catalytic subunit & 2.13 & 2.09 \\
Bcat2 & branched chain aminotransferase 2, mitochondrial & 2.07 & 0.018 \\
Aldh1a3 & aldehyde dehydrogenase 1 family, member A3 & 2.05 & 0.004 \\
Ptgis & prostaglandin I2 (prostacyclin) synthase & 2.01 & 0.05 \\
Ptges & prostaglandin E synthase & -2.04 & 0.016 \\
Ckmt1 & creatine kinase, mitochondrial 1, ubiquitous & -2.10 & 0.05 \\
Ptgs2 & prostaglandin-endoperoxide synthase 2 & -3.21 & -3.35 \\
\hline
\end{tabular}

doi:10.1371/journal.pone.0053831.t003

and 2). Csf2 has been known to exert anti-apoptotic activity through the expression of Bcl-2 family proteins in neural progenitor cells via JAK/STAT5-Bcl-2 pathway [23]. Csf2 also abrogates ischemia and thus prevents cardiomyocyte death by neovascularization [24]. IL-6 activates JAK/STAT3 and phosphatidylinositol 3-kinase (PI3K) pathways, thereby promoting cardiomyocyte survival [27,28]. Conversely, Axin2, Msx2, Calr and PKC $\varepsilon$ that mediate inhibition of cell proliferation and survival were up-regulated. Axin2, a most highly up-regulated gene (12.17fold), is a negative regulator of the Wnt signaling pathway that promotes the phosphorylation and degradation of $\beta$-catenin, resulting in cardomyocyte survival and hypertrophy [31,57]. PKCE can phosphorylate insulin receptor substrates (IRS) on serine residues impairing activation of PI3K in response to insulin $[33,58]$. Loss of PKC $\varepsilon$ selectively impairs signaling through the Bcell receptor, resulting in inhibition of cell proliferation and survival, as well as defects in the activation of ERK and the transcription of NF- $\mathrm{KB}-$ dependent genes [59]. Consistent with these data, our results showed that increased expression of atrogin-1 significantly promoted cardiomyocyte apoptosis under basal condition or in response to LPS stimulation (Figure 4).

Table 4. GO:0048468 cell development and hypertrophy.

\begin{tabular}{|c|c|c|c|}
\hline Gene Symbol & Gene Title & Fold change & $P$ value \\
\hline Krt19 & keratin 19 & 13.9 & 0.0035 \\
\hline Bhlha15 & basic helix-loop-helix family, member a15 & 4.27 & $1.1 \mathrm{E}-04$ \\
\hline Calr & calreticulin & 3.05 & $1.2 \mathrm{E}-04$ \\
\hline Lef1 & lymphoid enhancer binding factor 1 & 2.98 & 1.7E-04 \\
\hline Csrp2 & cysteine and glycine-rich protein 2 & 2.36 & 0.0021 \\
\hline Ccnd2 & cyclin D2 & 2.21 & 0.0067 \\
\hline Eif2ak3 & eukaryotic translation initiation factor 2 alpha kinase 3 & 2.14 & $3.1 \mathrm{E}-04$ \\
\hline Hook1 & hook homolog 1 (Drosophila) & 2.13 & $6.3 \mathrm{E}-04$ \\
\hline Xbp1 & X-box binding protein 1 & 2.07 & 4.1E-05 \\
\hline Lppr4 & plasticity related gene 1 & 2.05 & 0.018 \\
\hline Cdk5rap3 & CDK5 regulatory subunit associated protein 3 & 2.03 & 0.0021 \\
\hline Myh6 & myosin, heavy chain 6 , cardiac muscle, alpha & 2.02 & 0.05 \\
\hline Mapt & microtubule-associated protein tau & -2.17 & 0.027 \\
\hline Itga3 & Integrin alpha 3 & -2.18 & $1.6 \mathrm{E}-05$ \\
\hline Foxa2 & forkhead box A2 & -5.28 & $2.0 \mathrm{E}-05$ \\
\hline
\end{tabular}


Table 5. GO:0006950 response to inflammation.

\begin{tabular}{|c|c|c|c|}
\hline Gene Symbol & Gene Title & Fold change & $P$ value \\
\hline Dnajc3 & DnaJ (Hsp40) homolog, subfamily C, member 3 & 6.77 & 4.2E-05 \\
\hline Rab27a & Similar to RIKEN CDNA 1110059 E24 & 5.69 & 0.0011 \\
\hline Hyou1 & hypoxia up-regulated 1 & 5.48 & 5.7E-06 \\
\hline |l11r1 & interleukin 1 receptor, type I & 4.16 & 0.001 \\
\hline Armet & arginine-rich, mutated in early stage tumors & 3.81 & $1.1 \mathrm{E}-04$ \\
\hline Herpud1 & homocysteine-inducible, endoplasmic reticulum stress-inducible, ubiquitin-like $d$ & 3.46 & $5.7 \mathrm{E}-05$ \\
\hline Dnajb9 & DnaJ (Hsp40) homolog, subfamily B, member 9 & 3.27 & $6.1 \mathrm{E}-05$ \\
\hline Hspa5 & heat shock protein 5 & 3.19 & $2.8 \mathrm{E}-04$ \\
\hline Pdia2 & protein disulfide isomerase famil & 2.33 & 0.004 \\
\hline Sels & selenoprotein $\mathrm{S}$ & 2.29 & $1.5 \mathrm{E}-04$ \\
\hline Eif2ak3 & eukaryotic translation initiation factor 2 alpha kinase 3 & 2.15 & $3.1 \mathrm{E}-04$ \\
\hline Hspb7 & heat shock protein family, member 7 (cardiovascular) & 2.12 & $3.8 \mathrm{E}-04$ \\
\hline Xbp1 & X-box binding protein 1 & 2.07 & 4.1E-05 \\
\hline Gclc & glutamate-cysteine ligase, catalytic subunit & 2.05 & 0.018 \\
\hline Txndc4 & thioredoxin domain containing 4 (endoplasmic reticulum) & 2.03 & 7.4E-05 \\
\hline Serp1 & stress-associated endoplasmic reticulum protein 1 & 2.02 & 0.0019 \\
\hline Psen2 & presenilin 2 & -2.03 & $7.8 \mathrm{E}-04$ \\
\hline Lbp & lipopolysaccharide binding protein & -2.10 & 0.05 \\
\hline Ptgis & prostaglandin 12 (prostacyclin) synthase & -2.10 & 0.016 \\
\hline $\mathrm{Cxcl} 3$ & chemokine (C-X-C motif) ligand 3 & -2.13 & 0.05 \\
\hline Asf1a & ASF1 anti-silencing function 1 homolog A (S. cerevisiae) & -2.18 & $4.0 \mathrm{E}-4$ \\
\hline Cxcl6 & chemokine (C-X-C motif) ligand 6 & -2.25 & 0.045 \\
\hline Ptges & prostaglandin E synthase & -2.25 & 0.05 \\
\hline Cadm1 & cell adhesion molecule 1 & -2.34 & 0.0026 \\
\hline Tfpi2 & tissue factor pathway inhibitor 2 & -2.37 & 0.05 \\
\hline $\mathrm{Hpx}$ & hemopexin & -2.45 & 0.02 \\
\hline Ptger3 & Prostaglandin E receptor 3 (subtype EP3) & -2.50 & 0.016 \\
\hline Cxcl1 & chemokine (C-X-C motif) ligand 1 & -2.50 & 0.016 \\
\hline $\mathrm{Cxcl} 2$ & chemokine (C-X-C motif) ligand 2 & -2.60 & 0.05 \\
\hline Reg3g & regenerating islet-derived 3 gamma & -2.60 & 0.05 \\
\hline $\mathrm{Ccl} 20$ & chemokine (C-C motif) ligand 20 & -3.17 & 0.006 \\
\hline Ptgs2 & prostaglandin-endoperoxide synthase 2 & -3.56 & 0.02 \\
\hline $11-6$ & interleukin 6 & -4.83 & 0.006 \\
\hline Serpinb2 & serine (or cysteine) peptidase inhibitor, clade B, member 2 & -5.45 & 0.017 \\
\hline
\end{tabular}

Hypertrophy is a major contributor to cardiac dysfunction in human. Alterations of cardiac gene expression are central to ventricular dysfunction in human $\mathrm{HF}$ [5]. Our previous studies and others have demonstrated that atrogin-1 plays a crucial role in skeletal and cardiac muscle plasticity in response to hypertrophic or atrophic stimuli $[5,6,10,18]$. Accumulating evidences indicate that atrogin-1-dependent proteolysis of its substrates such as calcineurin, FOXOs, MyoD and eIF3-f could constitute the important events to regulate myocyte growth and hypertrophy $[5,6,17,19]$. However, the detailed molecular mechanisms by which atrogin- 1 contributes to hypertrophy via transcription remain to be elucidated. The present results showed that many genes significantly altered in cell development and hypertrophic cardiomyopathy by overexpressed atrogin-1, Myh6, Serca2 (ATP2A2) and Prkcz were significantly up-regulated, whereas Itga3 (also known as CD49c) and Foxa2 were down-
regulated.(Table 4). With respect to hypertrophic cardiomyopathy, Serca2 and Myh6 are major determinants of both cardiac relaxation and contraction. The Serca2 is of central importance for refilling of the sarcoplasmic reticulum (SR) $\mathrm{Ca}_{2}^{+}$store and cardiac contractility. Deletion of Serca2 function is associated with $\mathrm{HF}$ in animal models [39], whereas lentivirus vector-mediated Serca2 gene transfer ameliorates $\mathrm{HF}$ induced by myocardial infarction in rat [40]. In the heart, two isoforms of myosin heavy chain (MHC), Myh6 (also known as $\alpha-\mathrm{MHC}$ ) and Myh7 (also known as $\beta-\mathrm{MHC}$ ), exist in the mammalian ventricular myocardium, and $\alpha$-MHC is highly expressed in adult cardiomyocytes, whereas $\beta$-MHC is expressed in embryonic cardiomyocytes. Cardiac stress triggers adult hearts to undergo hypertrophy and a shift from Myh6 to fetal Myh7 expression. Furthermore, LPS is known to directly induce cardiomyocyte hypertrophy through activation of calcineurin signaling pathway [49], whereas overex- 
A
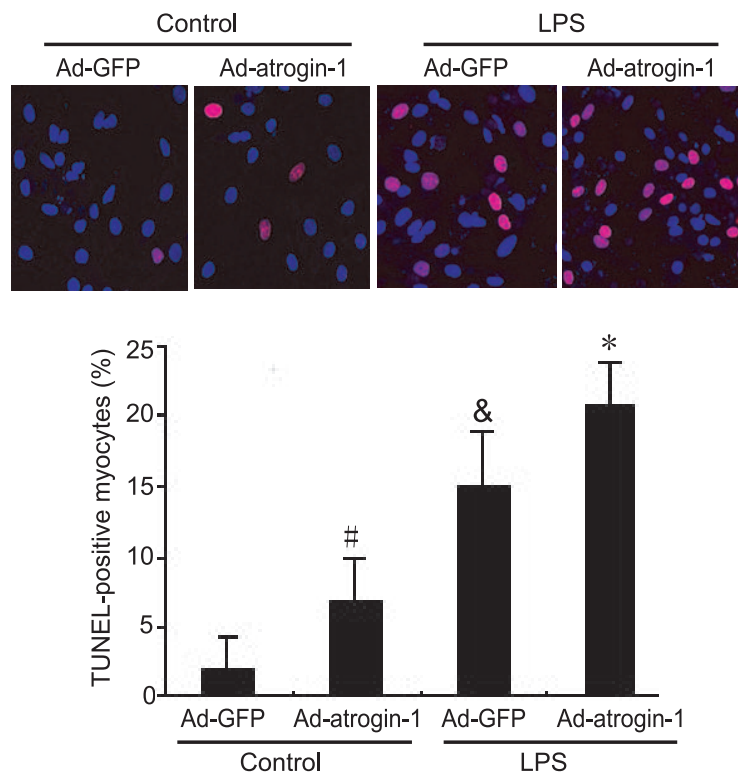

$\mathrm{B}$
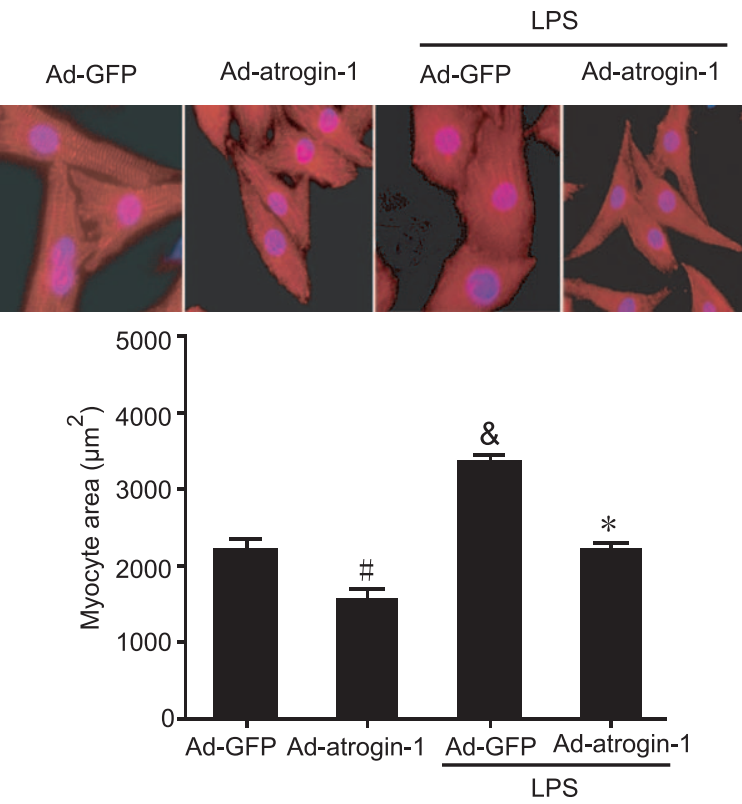

C

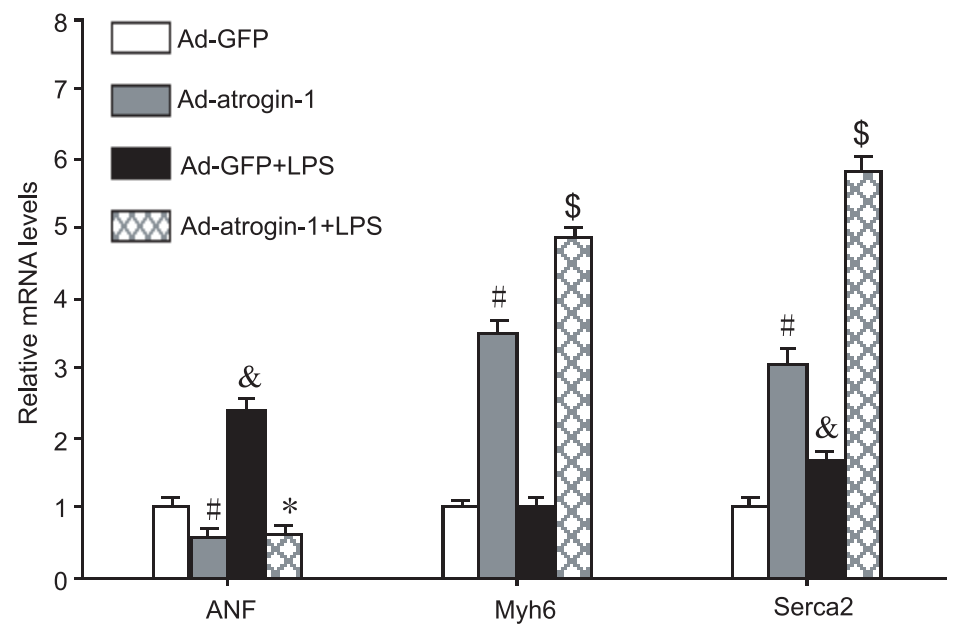

D

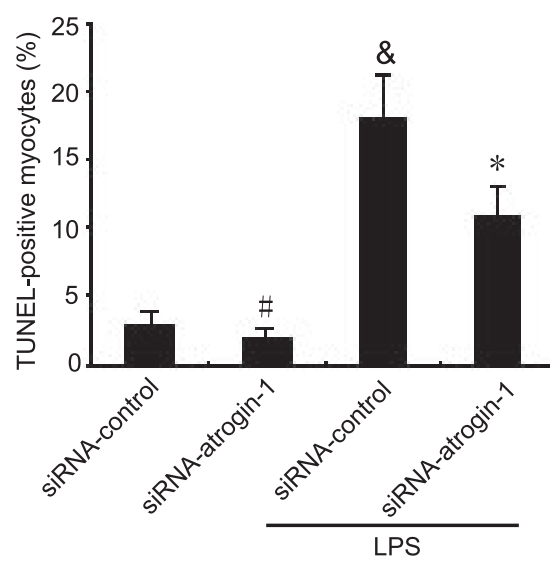

$E$

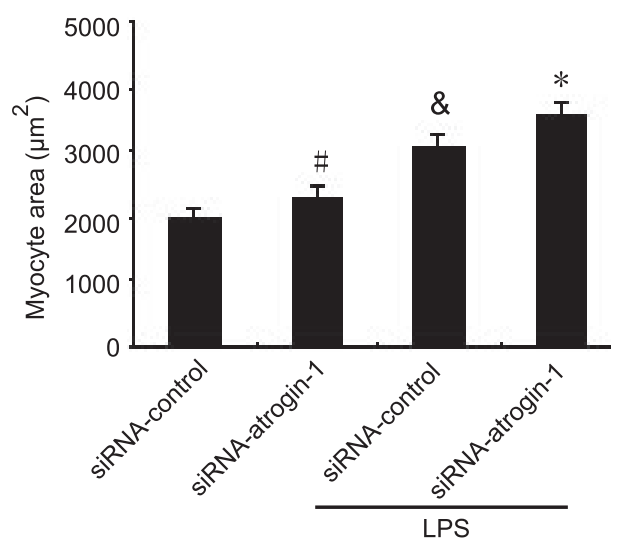

Figure 4. Effects of atrogin-1 overexpression on cardiomyocyte apoptosis and hypertrophy. Neonatal rat cardiomyocytes were infected with Ad-GFP or Ad-atrogin-1-GFP for $24 \mathrm{~h}$ and then treated with LPS $(1 \mu \mathrm{g} / \mathrm{ml})$ for additional 24 hours. A. Apoptosis was detected and quantified using TUNEL assay (red), and nuclei were counterstained with DAPI (blue). A representative field is shown for each condition (top panels), 
Magnification, $\times 400$. Quantitative analysis of TUNEL-positive cells from three independent experiments (bottom panels). B. The cells were fixed and stained with anti- $\alpha$-actinin antibody followed by Alexa Fluor 568 -conjugated goat anti-mouse lgG (red), and nuclei were stained with DAPI (blue). A representative field is shown for each condition (top panels), Magnification, $\times 200$. Quantitative analysis of cell surface area (a minimum of 100 randomly chosen cells measured in each group) (bottom panels). Data represent the mean \pm SEM $(n=3) .{ }^{\#} P<0.05,{ }^{*} P<0.01$ vs. Ad-GFP; ${ }^{*} P<0.05$; ${ }^{\$} P<0.01$ vs. Ad-GFP+LPS. C. The qRT-PCR analysis of ANF, Myh6 and serca2 mRNA expression was performed in triplicate using specific oligonucleotides primers. D and E. Neonatal rat cardiomyocytes were infected with Ad-siRNA-atrogin-1 or Ad-siRNA-control for 24 hours. Analysis of apoptosis and cell surface area were performed as in $A$ and $B$. Data represent the mean \pm SEM $(n=3)$. ${ }^{\#} P<0.05,{ }^{*} P<0.01$ vs. siRNA-control; ${ }^{*} P<0.05$ vs. siRNA-control +LPS.

doi:10.1371/journal.pone.0053831.g004

pression of atrogin-1 promotes calcineurin degradation and inhibits its activity, resulting in inhibition of cardiac hypertrophy [5]. Consistent with our previous data [5,6], the present study showed that overexpression of atrogin-1 inhibits LPS-induced cardiomyocyte hypertrophy, decreased the expression of hypertrophic marker ANF and up-regulated the level of Myh6 expression in cardiomyocytes. Thus, these results further confirmed that atrogin1 exerts an important role in controlling cardiomyocyte hypertrophy.

Functional annotation of the atrogin-1-infected cardiomyocyte signature analysis led us to three main pathways: metabolic pathways, inflammation signaling pathways and hypertrophic cardiomyopathy. There is a substantial body of work linking metabolic and inflammatory pathways with cardiovascular diseases including hypertrophy and HF [60,61]. Changes in mitochondrial gene expression were evident, ranging from the up-regulation of genes involved in energy metabolism, such as fatty acid biosynthetic process and ATP synthesis, and those important in maintaining energy metabolic pathways, such as Ptgis, Pycrl and Lsynal, SLC33Al (Table 5). Ptgis mediates prostaglandin biosynthetic process and fatty acid biosynthetic process $[34,62]$. Pycrl encodes an enzyme involved in the cellular response to oxidative stress and amino acid biosynthetic process [35]. The Pycrl protein is located in the mitochondria-the "power houses" of the cell that provide energy for cell's consumption [35]. Lsynal encodes a rate-limiting enzyme in the synthesis of all inositolcontaining compounds [63]. SLC33Al functions as an acetyl-CoA transporter that is required for the formation of $\mathrm{O}$-acetylated (Ac) gangliosides $[64,65]$. These data indicate that atrogin-1 plays an important role in regulating cardiomyocyte metabolic pathway.

It is well known that LPS directly induces inflammation in various cell types $[1,2]$. We then determine if atrogin- 1 inhibits LPS-induced proinflammatory response in cardiomyocytes. Atrogin-1 overexpression markedly inhibited the expression of proinflammatory-related genes including IL-1 $\beta$, IL-6, Lbp, Ptgs2 and Serpinb2, Cxcl1, Cxcl2 and Ccl20 in cardiomyocytes, but increased anti-inflammatory gene IL-1rl expression compared to Ad-GFP control under basal condition or in response to LPS stimulation (Figure 5A, Table 5), whereas these effects were reversed by knockdown of atrogin- 1 by siRNA (Figure 5B, Table 5). These results indicate that increased expression of atrogin- 1 inhibits cardiomyocyte inflammation.

The MAPK and NF- $\mathrm{BB}$ signaling pathways have been identified as the crucial regulators of cardiomyocyte apoptosis, cardiac hypertrophy and inflammation in response to various injury [66], but it is unknown if atrogin-1 affects activation of MAPK and NF$\kappa \mathrm{B}$ signal pathways in cardiomyocytes in response to LPS. Recently, a study demonstrated that depletion of atrogin- 1 inhibits cardiac hypertrophy in part through stabilization of I $\mathrm{I} B-\alpha$ and inactivation of NF- $\mathrm{BB}$ [12]. To further investigate the mechanisms of atrogin-1 in LPS-induced cardiac injury, we examined activation of ERK, JNK, p38 and p65/NF- $\mathrm{KB}$ signaling pathways. Under basal condition, overexpression of atrogin-1 decreased the levels of ERK, JNK, p38 and p65/NF- $\mathrm{B}$ phosphorylation compared with
Ad-GFP control (Figure 6A), suggesting that atrogin-1 may be involved in regulation of MAPK and NF- $\kappa \mathrm{B}$ activation. After stimulation of LPS, the levels of ERK, JNK, p38 and p65/NF-кB phosphorylation were markedly increased, whereas these effects were markedly attenuated by atrogin-1 infection in cardiomyocytes (Figure 6A). In contrast, knockdown of endogenous atrogin-1 in cardiomyocytes had opposite effects (Figure 6B). Thus, atrogin-1 exerts its inhibitory effects on cardiomycoyte survival, hypertrophy and inflammation via inhibition of MAPKs and NF- $\kappa B$ pathways.

\section{Conclusion}

The current study provides a comprehensive, global view of gene expression patterns induced by atrogin- 1 in the neonatal rat cardiomyocytes. Overexpression of atrogin-1 results in marked alterations of gene expression profiles that are associated with cardiomyocyte survival, proliferation, inflammation, metabolism and hypertrophy, thereby leading to inhibition of cardiomycoyte survival, growth and hypertrophy under basal condition or in response to LPS stimualtion. The mechanisms underlying these effects were associated with inactivation of MAPK (ERK, JNK and p38) and NF- $\kappa \mathrm{B}$ signaling pathways. These results may provide novel insight into how atrogin-1 modulates the programming of cardiac muscle gene expression.

\section{Materials and Methods}

All procedures were approved by and performed in accordance with the Animal Care and Use Committee of Capital Medical University (20110820).

\section{Neonatal rat cardiomyocytes isolation and culture}

Primary cardiomyocytes were prepared by enzymatic disassociation of 1- to 2-day old Sprague-Dawley rats as described previously [5]. The isolated cardiomyocytes were resuspended in fresh DMEM/F12 containing 10\% fetal bovine serum (FBS) and $1 \%$ penicillin-streptomycin and were plated into the Laminin (Sigma) pre-coated dishes (Corning) and incubated for $24 \mathrm{~h}$ at $37^{\circ} \mathrm{C}$, then cultured with serum-free DMEM/F12 in later experiments.

\section{Adenovirus Infection}

Twenty-four hours after plating, neonatal rat cardiomyocytes were infected with various adenovirus vectors as indicated and cultured with serum-free DMEM/F12 for 24 hours. Recombinant adenoviruses expressing GFP alone (Ad-GFP), atrogin-1 (Ad-atrogin1-GFP), siRNA-control or siRNA-atrogin-1 were generated using the AdEasy system (MP Biomedicals Inc.) as described previously [18]. Fluorescent images were collected on a fluorescence microscope (Nikon TE 2000-U, Japan). The infection efficiency of adenoviruses was determined by counting the number of cells with green fluorescent protein (GFP). 


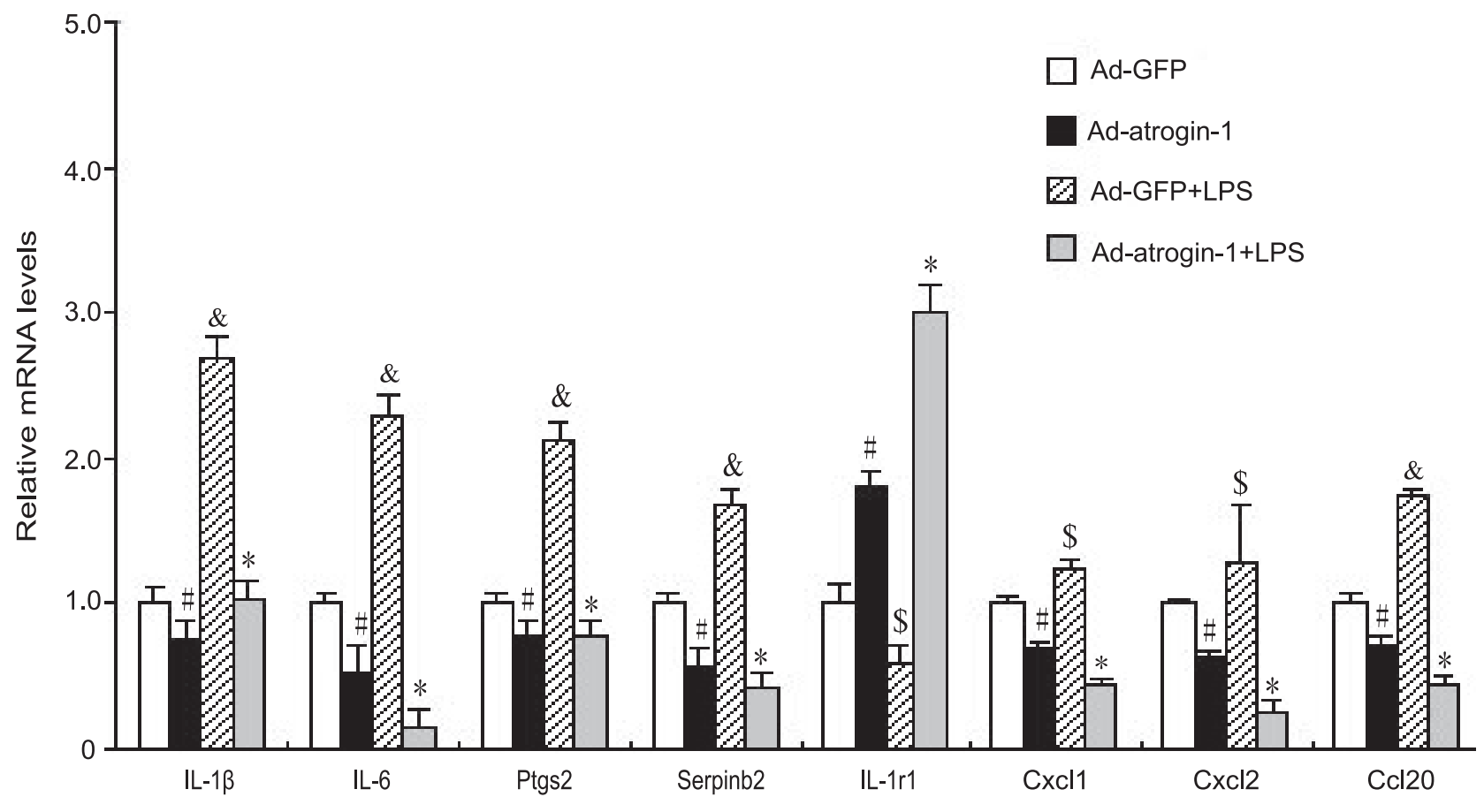

B

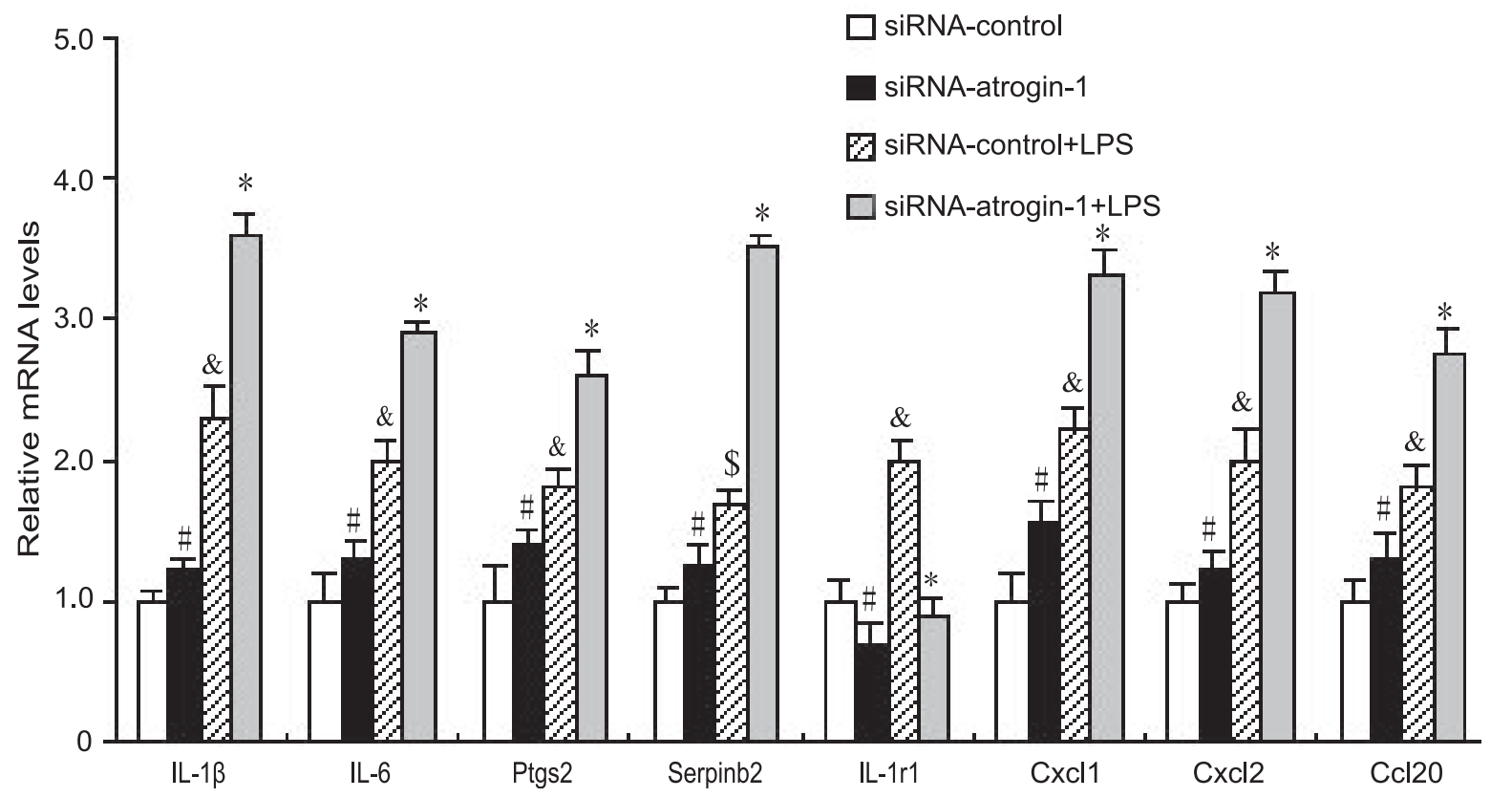

Figure 5. Effect of atrogin-1 on inflammation. A. Neonatal rat cardiomyocytes were infected with Ad-GFP or Ad-atrogin-1-GFP for $24 \mathrm{~h}$ and then treated with LPS $(1 \mu \mathrm{g} / \mathrm{ml})$ for additional 24 hours. The qRT-PCR analysis of gene expression was performed in triplicate using specific oligonucleotides primers. Data represent the mean \pm SEM. ${ }^{\#} P<0.05,{ }^{\&} P<0.01,{ }^{\$} P<0.05$ vs. Ad-GFP; ${ }^{*} P<0.01$ vs. Ad-GFP+LPS. B. Neonatal rat cardiomyocytes were infected with Ad-siRNA-control or Ad-siRNA-atrogin- 1 for $24 \mathrm{~h}$ and then treated with LPS $(1 \mu \mathrm{g} / \mathrm{ml})$ for additional $24 \mathrm{hours}$. The qRT-PCR analysis of gene expression was performed as in A. Data represent the mean \pm SEM. ${ }^{\#} P<0.05,{ }^{*} P<0.01,{ }^{5} P<0.05$ vs. siRNA-control; ${ }^{*} P<0.01$ vs. Ad-siRNA+LPS.

doi:10.1371/journal.pone.0053831.g005

\section{RNA isolation and GeneChip processing}

Total RNA was isolated with TRIzol (Invitrogen) from neonatal rat cardiomyocytes in three independent experiments according to manufacturer's instructions. The quantity and purity of all RNA samples was determined using the Nanodrop ND-1000 spectrophotometer (Thermo Fisher, Waltham, MA, US), and RNA integrity was determined with the Bioanalyzer 2100 (Agilent technologies, Santa Clara, CA, US). Five micrograms of total 
A
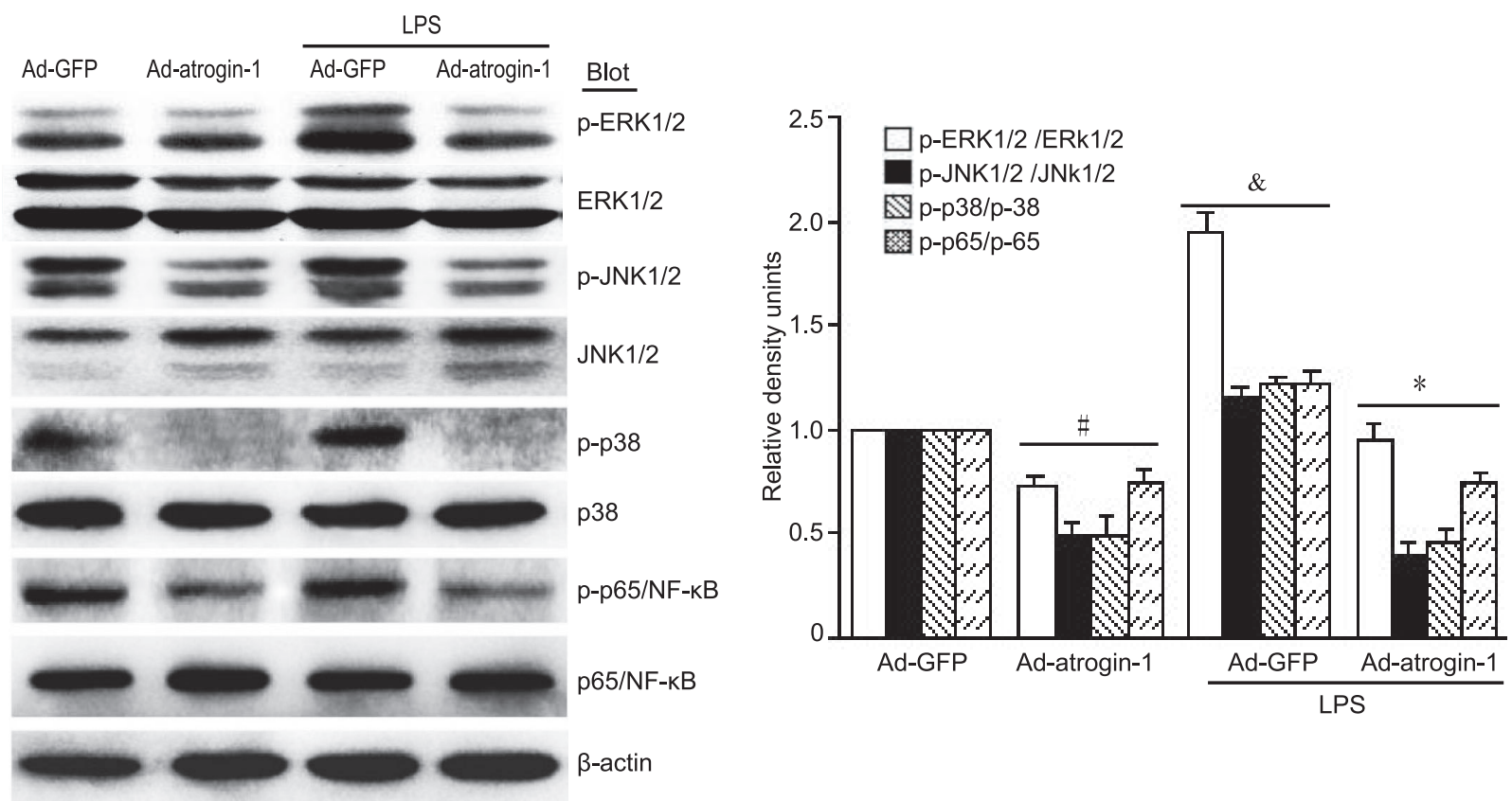

B
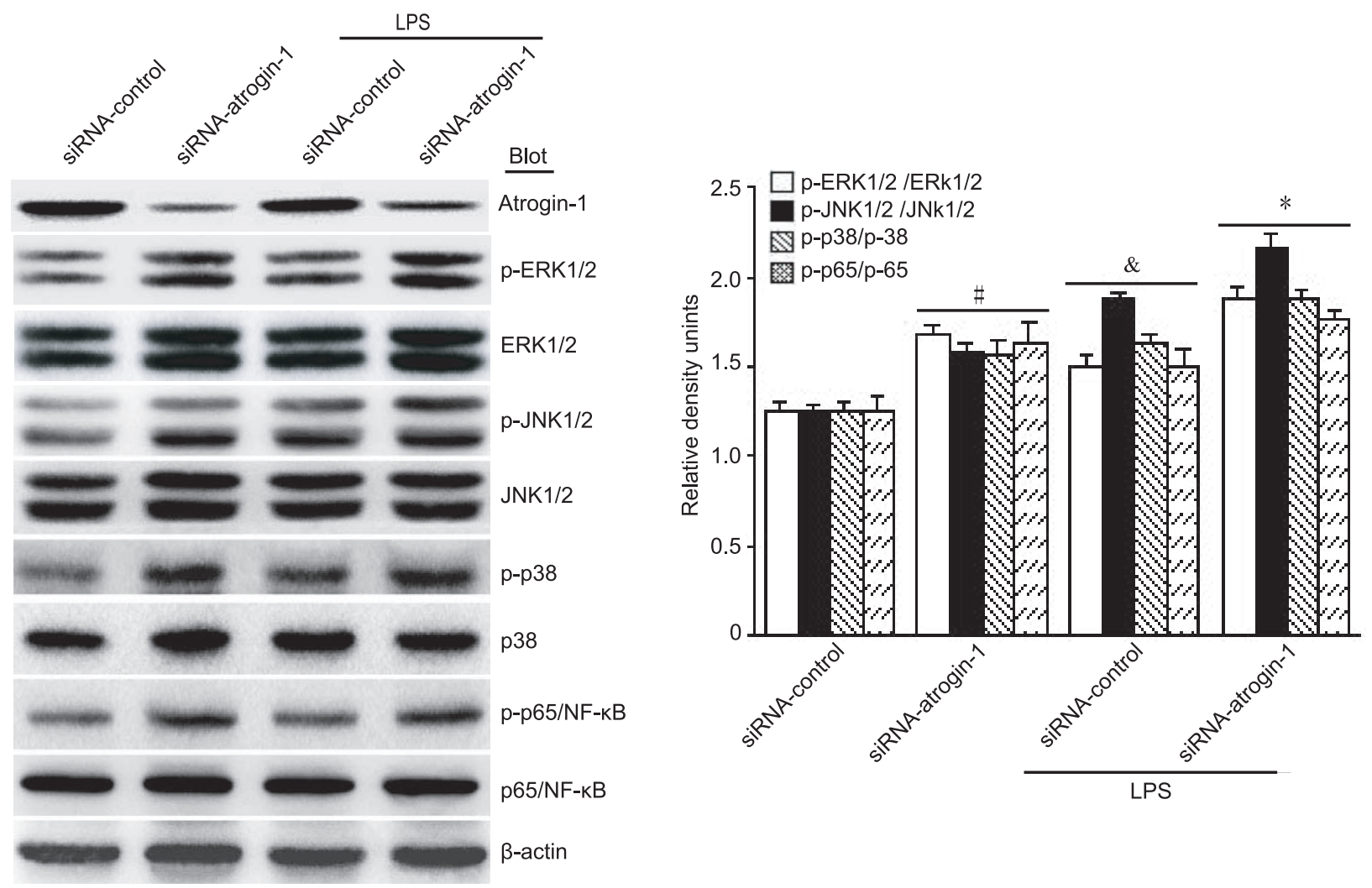

Figure 6. Effects of atrogin-1 on MAPK and NF-KB signaling pathways. A. Neonatal rat cardiomyocytes were infected with Ad-GFP or Adatrogin-1-GFP for $24 \mathrm{~h}$ and then treated with LPS $(1 \mu \mathrm{g} / \mathrm{ml})$. The protein levels of total and phospho-ERK1/2, JNK1/2, p38 and p65/NF-kB were detected by Western blot analysis (left panels). Quantitative analysis of relative intensity of phosphorylated proteins was shown (right panel). Data represent the mean \pm SEM $(n=3)$. ${ }^{*} P<0.05,{ }^{*} P<0.05$ vs. Ad-GFP; ${ }^{*} P<0.01$ vs. Ad-GFP+LPS. B. Neonatal rat cardiomyocytes were infected with adenovirus siRNA-control or siRNA-atrogin-1 and then treated with LPS $(1 \mu \mathrm{g} / \mathrm{ml})$. The protein levels were detected as in A (left panels). Quantitative analysis of relative intensity of phosphorylated proteins was shown (right panel). Data represent the mean \pm SEM $(n=3)$. \# $P<0.05,{ }^{\&} p<0.05$ vs. siRNA-control; $* P<0.05$ vs. siRNA-control+LPS.

doi:10.1371/journal.pone.0053831.g006 
Table 6. Primers used for Quantitative realtime RT-PCR Analyses.

\begin{tabular}{|c|c|c|}
\hline Genes & Forward primer & Reverse primer \\
\hline Axin2 & 5'-GCGTGAGATCCACAGAAACG-3' & 5'-TCGCTGGATAACTCGCTGTC-3' \\
\hline Calr & 5'-CCGATGCGAATATCTATGCC-3' & 5'-TCATTGCCAAACTCCTCTGC-3' \\
\hline Dkk2 & 5'-CATGAACCAAGGACTGGCTT-3' & 5'-CAGGCTGAAGATCCTTGGTG-3' \\
\hline$\| 1 \mathrm{r} 1$ & 5'-TTGTCTCATTGTGCCTCTGC-3' & 5'-TAAGAGGACAGCTGCGAATG-3' \\
\hline CyclinD2 & 5'-TCAAGTGCGTGCAGAAGGAC-3' & $5^{\prime}-$ TGGCCAGAGGAAAGACCTCT -3' \\
\hline MMP3 & 5'-TCCTTCGATGCAGTCAGCAC-3' & 5'-TGTTGGATGGAAGAGACGGC-3' \\
\hline Cadm1 & 5'-CACAGGTGATGGGCAGAATC-3' & 5'-TGCCTGTTGGGGTTCAGTAG-3' \\
\hline Cxxl1 & 5'-CCACACTCAAGAATGGTCGC-3' & 5'-GTTCACCAGACAGACGCCAT-3' \\
\hline $\mathrm{Cxcl} 2$ & 5'-TTTGTCTCAACCCTGAAGCC-3' & 5'-TGAGGTACAGGAGCCCATGT-3' \\
\hline Cxcl6 & 5'-TCCTGCTCGTCATTCACCCT-3' & 5'-CAAACACAGCGTAGCTCCGT-3' \\
\hline II-6 & 5'-TCTGCTCTGGTCTTCTGGAG-3' & 5'-TTGCTCTGAATGACTCTGGC-3' \\
\hline$\|-1 \beta$ & 5'-CTCTGTGACTCGTGGGATGATG-3' & 5'-CCACTTGTTGGCTTATGTTCTGTC-3' \\
\hline Ptgs2 & 5'-GATCACATTTGATTGACAGC-3' & 5'-ТССТТАТТССТTСАСАCC-3' \\
\hline Serpinb2 & 5'-TCCTTGGTGCTCAGGCTAAC-3' & 5'-CAGCCATGGAAGTTCTCTGG-3' \\
\hline $\mathrm{Ccl} 20$ & 5'-ACCTCCTCAGCCTAAGAACCAAGA-3' & $5^{\prime}$-TGTGCAGTGATGTGCAGGTGAAAC-3' \\
\hline Myh7 & 5'-CGAGGCAAGCTCACGTATAC-3' & 5'-CTTGGCTTCTGTTTCCTCCT-3' \\
\hline ANF & 5'-CTTCTCCATCACCAAGGGCTT-3' & 5'-GGATTTGCTCCAATATGGCCT-3' \\
\hline serca2 & 5'-CAGTTCATCCGCTACCTCATCTCC-3' & 5'-CGCAGTGGCAGGCAGACC-3' \\
\hline GADPH & 5'-CCCCCAATGTATCCGTTGTG & TAGCCCAGGATGCCCTTTAGT \\
\hline$\beta$-actin & 5'-GGAGATTACTGCCCTGGCTCCTA-3' & 5'-GACTCATCGTACTCCTGCTTGCTG-3' \\
\hline
\end{tabular}

RNA was amplified with the GeneChip One-Cycle cDNA synthesis Kit (Affymetrix) and GeneChip IVT labeling Kit (Affymetrix). Fifteen micrograms biotin-labeled complementary RNA (cRNA) was fractionated and hybridized to Affymetrix GeneChip Rat Genome 2302.0 array according to the manufacturer's instructions. After 16 h of hybridization, the Gene Chips were washed and stained on a Fluidics Station 450 (Affymetrix) and scanned in a confocal scanner (Affymetrix GeneChip Scanner 3000) according to the Affymetrix GeneChip Expression Analysis Manual as described previously [67]. On the GeneChip Rat Genome 2302.0 array, 31,000 probe sets analyze the expression of 30,000 transcripts representing 28,000 known genes.

\section{Microarray data analysis}

Analysis of microarray data was performed as described previously [67]. Briefly, raw intensities from the CEL files were analyzed using GeneSpring GX 11.0 (Agilent Technologies) to generate robust multi-array average (RMA) intensity in $\log _{2}$ scale for each probe set. Differentially expressed genes in Ad-atrogin-1-GFP group compared to Ad-GFP control group were detected with two statistical tests, a statistical technique used to compare means of two samples implemented in GeneSpring GX 11.0. For each transcript, fold change, statistical significance of differential expression and false discovery rate (FDR) were calculated. Fold change was calculated using the average signal from each experimental group. The recovered P-values of the comparisons were then corrected using a step-up false negative/positive rate value of $5 \%$. The resulting list of significantly differentially expressed genes was filtered to include only genes that demonstrated 2-fold or greater up- or down-regulation. To search for enrichment of specific biological processes, the genes showing significantly differential expression between the two groups were classified into functional groups according to GO (Gene
Ontology). As input, the differentially regulated probe sets from the comparison were used. As output, significant processes (GOTERM) with fold changes $\geq 2$-fold or $\leq-2$-fold were generated. Furthermore, pathway analysis for the comparison was conducted through the use of KEGG Pathways database, which is a bioinformatics resource for linking genomes to life and the environment. Details can be found at http://www.genome.jp/kegg/. The microarray data discussed in this publication have been deposited in NCBI's Gene Expression Omnibus and are accessible through GEO Series accession number GSE31117 (http://www.ncbi.nlm.nih.gov/geo/ query/acc.cgi?acc $=$ GSE31117).

\section{Real-time quantitative PCR analysis}

Microarray results for the expression profiling experiments were verified by real-time quantitative RT-PCR (qRT-PCR) using a Bio-Rad iQ5 Real-Time PCR detection system, employing $\beta$-actin as the endogenous control gene as described previously [67] . All reactions were conducted in triplicates and the data was analyzed using the delta delta $\mathrm{Ct}(\boldsymbol{\Delta} \mathbf{\Delta} \mathrm{C} t)$ method [68]. Oligonucleotide primers for qRT-PCR were designed using the Primer 5.0 software and the primers were designed to span large introns to eliminate possible contaminating genomic DNA. Rat gene-specific oligonucleotide primers used for qRT-PGR analyses are listed in Table 6 .

\section{Western blot analysis}

Cardiomyocytes were lysed in lysis buffer $(50 \mathrm{mM}$ Tris-HCl, pH 7.5, 1 mM DTT, 150 mM NaCl, 0.5\% NP-40, 1 mM EDTA, $1 \mathrm{mM}$ PMSF, $1 \mathrm{mM}$ Na3VO4, $1 \mathrm{mM} \mathrm{NaF}$, plus protease inhibitor cocktail). Forty micrograms of total proteins was separated by SDS-PAGE, transferred to PVDF membranes (Millipore), and analyzed by western blot with anti-atrogin-1 antibody (H300, Santa Cruz) as described previously [5], using $\beta$ - 
actin (I-19, Santa Cruz) as the internal control. Protein levels were quantified by using Gel-pro 4.5 Analyzer (Media Cybernetics).

\section{TUNEL assays}

Primary cardiomyocytes were infected with Ad-atrogin-1 or AdGFP control $(\mathrm{MOI}=10)$ for 24 hours and then treated with $10 \mathrm{ng} / \mathrm{ml}$ LPS (Sigma) for 24 hours. TUNEL assays were performed using the In Situ Cell Death Detection Kit, TMR red (Roche) according to manufacturer's instructions. Apoptosis was evaluated by Laser scanning confocal fluorescence microscope (Leica TCS SP2, Germany). Quantitation of cardiomyocyte apoptosis was performed based on the percentage of TMR red labeled nuclei with image-pro plus 6.0 software.

\section{Measurement of cardiomyocyte hypertrophy}

Primary cardiomyocytes were infected with Ad-atrogin-1 or AdGFP control $(\mathrm{MOI}=10)$ for 24 hours and then treated with PBS or lipopolysaccharides (LPS, $1 \mu \mathrm{g} / \mathrm{ml}$ ) for 8 hours, then cells were fixed and stained with anti- $\alpha$-actinin antibody followed by Alexa

\section{References}

1. Alexander C, Rietschel ET (2001) Bacterial lipopolysaccharides and innate immunity. Journal of endotoxin research 7:167-202.

2. Frantz S, Kobzik L, Kim YD, Fukazawa R, Medzhitov R, et al. (1999) Toll4 (TLR4) expression in cardiac myocytes in normal and failing myocardium. The Journal of clinical investigation 104:271-280.

3. Suzuki J, Bayna E, Li HL, Molle ED, Lew WY (2007) Lipopolysaccharide activates calcineurin in ventricular myocytes. Journal of the American College of Cardiology 49:491-499.

4. Willis MS, Townley-Tilson WH, Kang EY, Homeister JW, Patterson C (2010) Sent to destroy: the ubiquitin proteasome system regulates cell signaling and protein quality control in cardiovascular development and disease. Circ Res 106:463-478.

5. Li HH, Kedar V, Zhang C, McDonough H, Arya R, et al. (2004) Atrogin-1/ muscle atrophy F-box inhibits calcineurin-dependent cardiac hypertrophy by participating in an SCF ubiquitin ligase complex. The Journal of clinical investigation 114:1058-1071.

6. Li HH, Willis MS, Lockyer P, Miller N, McDonough H, et al. (2007) Atrogin-1 inhibits Akt-dependent cardiac hypertrophy in mice via ubiquitin-dependent coactivation of Forkhead proteins. The Journal of clinical investigation 117:3211-3223.

7. Zhang Y, Kang YM, Tian C, Zeng Y, Jia LX, et al. (2011) Overexpression of Nrdpl in the heart exacerbates doxorubicin-induced cardiac dysfunction in mice. PloS one 6:e21104

8. Adams V, Linke A, Wisloff U, Doring C, Erbs S, et al. (2007) Myocardial expression of Murf- 1 and MAFbx after induction of chronic heart failure: Effect on myocardial contractility. Cardiovasc Res 73:120-129.

9. Gomes MD, Lecker SH, Jagoe RT, Navon A, Goldberg AL (2001) Atrogin-1, a muscle-specific F-box protein highly expressed during muscle atrophy. Proc Natl Acad Sci U S A 98:14440-14445.

10. Bodine SC, Latres E, Baumhueter S, Lai VK, Nunez L, et al. (2001) Identification of ubiquitin ligases required for skeletal muscle atrophy. Science 294:1704-1708.

11. Arya R, Kedar V, Hwang JR, McDonough H, Li HH, et al. (2004) Muscle ring finger protein-1 inhibits PKC\{epsilon\} activation and prevents cardiomyocyte hypertrophy. The Journal of cell biology 167:1147-1159.

12. Usui S, Maejima Y, Pain J, Hong C, Cho J, et al. (2011) Endogenous Muscle Atrophy F-Box Mediates Pressure Overload-Induced Cardiac Hypertrophy Through Regulation of Nuclear Factor-\{kappa\}B. Circ Res 109:161-171.

13. Glass DJ (2005) Skeletal muscle hypertrophy and atrophy signaling pathways. Int J Biochem Cell Biol 37:1974-1984.

14. Li YP, Chen Y, John J, Moylan J, Jin B, et al. (2005) TNF-alpha acts via p38 MAPK to stimulate expression of the ubiquitin ligase atroginl/MAFbx in skeletal muscle. Faseb J 19:362-370.

15. Sandri M, Sandri C, Gilbert A, Skurk C, Calabria E, et al. (2004) Foxo transcription factors induce the atrophy-related ubiquitin ligase atrogin-1 and cause skeletal muscle atrophy. Cell 117:399-412.

16. Csibi A, Leibovitch MP, Cornille K, Tintignac LA, Leibovitch SA (2009) MAFbx/Atrogin-1 controls the activity of the initiation factor eIF3-f in skeletal muscle atrophy by targeting multiple C-terminal lysines. J Biol Chem 284:44134421 .

17. Tintignac LA, Lagirand J, Batonnet S, Sirri V, Leibovitch MP, et al. (2005) Degradation of MyoD mediated by the SCF (MAFbx) ubiquitin ligase. J Biol Chem 280:2847-2856.

18. Xie P, Guo S, Fan Y, Zhang H, Gu D, et al. (2009) Atrogin-1/MAFbx enhances simulated ischemia/reperfusion-induced apoptosis in cardiomyocytes through
Fluor 568-conjugated goat anti-mouse IgG (red), and nuclei were stained with DAPI (blue). A representative field is shown for each condition. A minimum of 100 randomly chosen cells measured in each group. Quantitation of cell surface area was performed as described previously [5].

\section{Statistical analysis}

Data for qRT-PCR analyses were presented as means \pm SEM. Differences between groups were evaluated for statistical significance using a two-tailed Student's $t$ test with GraphPad Prism 5.0 or Stata/SE 10.0 softwares. $P$ values less than 0.05 were regarded as significant.

\section{Author Contributions}

Conceived and designed the experiments: HHL JD CST. Performed the experiments: YZ HXW SBG HY XJZ. Analyzed the data: YZ HXW HY QF. Contributed reagents/materials/analysis tools: HHL JD YZ QF. Wrote the paper: HHL JD.

degradation of MAPK phosphatase-1 and sustained JNK activation. J Biol Chem 284:5488-5496.

19. Lagirand-Cantaloube J, Offner N, Csibi A, Leibovitch MP, Batonnet-Pichon S, et al. (2008) The initiation factor eIF3-f is a major target for atrogin 1/MAFbx function in skeletal muscle atrophy. Embo J 27:1266-1276.

20. Skurk C, Izumiya Y, Maatz H, Razeghi P, Shiojima I, et al. (2005) The FOXO3a transcription factor regulates cardiac myocyte size downstream of AKT signaling. The Journal of biological chemistry 280:20814-20823.

21. Galasso G, De Rosa R, Piscione F, Iaccarino G, Vosa C, et al. (2010) Myocardial expression of FOXO3a-Atrogin-1 pathway in human heart failure. Eur J Heart Fail 12:1290-1296.

22. Eisen MB, Spellman PT, Brown PO, Botstein D (1998) Cluster analysis and display of genome-wide expression patterns. Proc Natl Acad Sci U S A 95:14863-14868.

23. Choi JK, Kim KH, Park H, Park SR, Choi BH (2011) Granulocyte macrophage-colony stimulating factor shows anti-apoptotic activity in neural progenitor cells via JAK/STAT5-Bcl-2 pathway. Apoptosis 16:127-134.

24. Kovacic JC, Muller DW, Graham RM (2007) Actions and therapeutic potential of G-CSF and GM-CSF in cardiovascular disease. J Mol Cell Cardiol 42:19-33.

25. Loureiro B, Oliveira LJ, Favoreto MG, Hansen PJ (2011) Colonv-stimulating Factor 2 Inhibits Induction of Apoptosis in the Bovine Preimplantation Embryo. Am J Reprod Immunol.

26. Liu Y, Li PK, Li C, Lin J (2010) Inhibition of STAT3 signaling blocks the antiapoptotic activity of IL-6 in human liver cancer cells. J Biol Chem 285:2742927439.

27. Fredj S, Bescond J, Louault C, Delwail A, Lecron JC, et al. (2005) Role of interleukin-6 in cardiomyocyte/cardiac fibroblast interactions during myocyte hypertrophy and fibroblast proliferation. J Cell Physiol 204:428-436.

28. Ancey C, Corbi P, Froger J, Delwail A, Wijdenes J, et al. (2002) Secretion of IL6, IL-11 and LIF by human cardiomyocytes in primary culture. Cytokine 18:199-205.

29. Moreb JS, Baker HV, Chang LJ, Amaya M, Lopez MC, et al. (2008) ALDH isozymes downregulation affects cell growth, cell motility and gene expression in lung cancer cells. Mol Cancer 7:87.

30. Wu LY, Li M, Hinton DR, Guo L, Jiang S, et al. (2003) Microphthalmia resulting from MSX2-induced apoptosis in the optic vesicle. Invest Ophthalmol Vis Sci 44:2404-2412.

31. Yu HM, Liu B, Costantini F, Hsu W. (2007) Impaired neural development caused by inducible expression of Axin in transgenic mice. Mech Dev 124:146156.

32. Baba Y, Nosho K, Shima K, Goessling W, Chan AT, et al. (2010) PTGER2 overexpression in colorectal cancer is associated with microsatellite instability, independent of $\mathrm{CpG}$ island methylator phenotype. Cancer Epidemiol Biomarkers Prev 19:822-831.

33. Lee SJ, Kim JY, Nogueiras R, Linares JF, Perez-Tilve D, et al. (2010) PKCzetaregulated inflammation in the nonhematopoietic compartment is critical for obesity-induced glucose intolerance. Cell Metab 12:65-77.

34. Nakayama T (2010) Genetic polymorphisms of prostacyclin synthase gene and cardiovascular disease. Int Angiol 29:33-42.

35. Reversade B, Escande-Beillard N, Dimopoulou A, Fischer B, Chng SC, et al. (2009) Mutations in PYCR1 cause cutis laxa with progeroid features. Nat Genet 41:1016-1021

36. Kwon S, Kim D, Rhee JW, Park JA, Kim DW, et al. (2010) ASB9 interacts with ubiquitous mitochondrial creatine kinase and inhibits mitochondrial function. BMC Biol 8:23. 
37. Carniel E, Taylor MR, Sinagra G, Di Lenarda A, Ku L, et al. (2005) Alphamyosin heavy chain: a sarcomeric gene associated with dilated and hypertrophic phenotypes of cardiomyopathy. Circulation 112:54-59.

38. Luo X, Shin DM, Wang X, Konieczny SF, Muallem S (2005) Aberrant localization of intracellular organelles, $\mathrm{Ca} 2+$ signaling, and exocytosis in Mist 1 null mice. J Biol Chem 280:12668-12675.

39. Andersson KB, Birkeland JA, Finsen AV, Louch WE, Sjaastad I, et al. (2009) Moderate heart dysfunction in mice with inducible cardiomyocyte-specific excision of the Serca2 gene. J Mol Cell Cardiol 47:180-187.

40. Niwano K, Arai M, Koitabashi N, Watanabe A, Ikeda Y, et al. (2008) Lentiviral vector-mediated SERCA2 gene transfer protects against heart failure and left ventricular remodeling after myocardial infarction in rats. Mol Ther 16:10261032.

41. Lantz KA, Vatamaniuk MZ, Brestelli JE, Friedman JR, Matschinsky FM, et al. (2004) Foxa2 regulates multiple pathways of insulin secretion. J Clin Invest 114:512-520.

42. Li M, Georgakopoulos D, Lu G, Hester L, Kass DA, et al. (2005) p38 MAP kinase mediates inflammatory cytokine induction in cardiomyocytes and extracellular matrix remodeling in heart. Circulation 111:2494-2502.

43. Tsuchiya H, Oka T, Nakamura K, Ichikawa A, Saper CB, et al. (2008) Prostaglandin E2 attenuates preoptic expression of GABAA receptors via EP3 receptors. J Biol Chem 283:11064-11071.

44. Heinzelmann M, Bosshart H (2005) Heparin binds to lipopolysaccharide (LPS)binding protein, facilitates the transfer of LPS to CD14, and enhances LPSinduced activation of peripheral blood monocytes. J Immunol 174:2280-2287.

45. Sun H, Xu B, Inoue H, Chen QM (2008) P38 MAPK mediates COX-2 gene expression by corticosterone in cardiomyocytes. Cell Signal 20:1952-1959.

46. Feng L (2000) Role of chemokines in inflammation and immunoregulation. Immunol Res 21:203-210.

47. Moser B, Willimann K. (2004) Chemokines: role in inflammation and immune surveillance. Ann Rheum Dis 63 Suppl 2:ii84-ii89.

48. Gonzalez-Navajas JM, Law J, Nguyen KP, Bhargava M, Corr MP, et al. (2010) Interleukin 1 receptor signaling regulates DUBA expression and facilitates Tolllike receptor 9-driven antiinflammatory cytokine production. J Exp Med 207:2799-2807.

49. Liu CJ, Cheng YC, Lee KW, Hsu HH, Chu CH, et al. (2008) Lipopolysaccharide induces cellular hypertrophy through calcineurin/NFAT-3 signaling pathway in H9c2 myocardiac cells. Molecular and cellular biochemistry 313:167-178.

50. Rose BA, Force T, Wang Y (2010) Mitogen-activated protein kinase signaling in the heart: angels versus demons in a heart-breaking tale. Physiol Rev 90:15071546 .

51. Jin B, Li YP (2007) Curcumin prevents lipopolysaccharide-induced atrogin-1/ MAFbx upregulation and muscle mass loss. Journal of cellular biochemistry 100:960-969.

52. Li YP, Lecker SH, Ghen Y, Waddell ID, Goldberg AL, et al. (2003) TNF-alpha increases ubiquitin-conjugating activity in skeletal muscle by up-regulating UbcH2/E220k. Faseb J 17:1048-1057.
53. Yamamoto Y, Hoshino Y, Ito T, Nariai T, Mohri T, et al. (2008) Atrogin-1 ubiquitin ligase is upregulated by doxorubicin via p38-MAP kinase in cardiac myocytes. Cardiovascular research 79:89-96.

54. Yoshida T, Semprun-Prieto L, Sukhanov S, Delafontaine P (2010) IGF-1 prevents ANG II-induced skeletal muscle atrophy via Akt- and Foxo-dependent inhibition of the ubiquitin ligase atrogin-1 expression. American journal of physiology 298:H1565-1570.

55. LiJJ, Zhang TP, Meng Y, Du J, Li HH (2011) Stability of F-box protein atrogin1 is regulated by p38 mitogen-activated protein kinase pathway in cardiac H9c2 cells. Cell Physiol Biochem 27:463-470.

56. Baines CP, Molkentin JD (2005) STRESS signaling pathways that modulate cardiac myocyte apoptosis. J Mol Cell Cardiol 38:47-62.

57. Jho EH, Zhang T, Domon C, Joo CK, Freund JN, et al. (2002) Wnt/betacatenin/Tcf signaling induces the transcription of Axin2, a negative regulator of the signaling pathway. Mol Cell Biol 22:1172-1183.

58. Lee S, Lynn EG, Kim JA, Quon MJ (2008) Protein kinase C-zeta phosphorylates insulin receptor substrate-1, -3 , and -4 but not -2 : isoform specific determinants of specificity in insulin signaling. Endocrinology 149:2451-2458.

59. Martin P, Duran A, Minguet S, Gaspar ML, Diaz-Meco MT, et al. (2002) Role of zeta PKC in B-cell signaling and function. Embo J 21:4049-4057.

60. Chen W, Frangogiannis NG (2010) The role of inflammatory and fibrogenic pathways in heart failure associated with aging. Heart failure reviews 15:415422.

61. Lusis AJ, Attie AD, Reue K.(2008) Metabolic syndrome: from epidemiology to systems biology. Nature reviews 9:819-830.

62. Gross S, Tilly P, Hentsch D, Vonesch JL, Fabre JE (2007) Vascular wallproduced prostaglandin E2 exacerbates arterial thrombosis and atherothrombosis through platelet EP3 receptors. J Exp Med 204:311-320.

63. Seelan RS, Lakshmanan J, Casanova MF, Parthasarathy RN (2009) Identification of myo-inositol-3-phosphate synthase isoforms: characterization, expression, and putative role of a 16-kDa gamma(c) isoform. J Biol Chem 284:94439457.

64. Kanamori A, Nakayama J, Fukuda MN, Stallcup WB, Sasaki K, et al. (1997) Expression cloning and characterization of a cDNA encoding a novel membrane protein required for the formation of $\mathrm{O}$-acetylated ganglioside: a putative acetylCoA transporter. Proc Natl Acad Sci U S A 94:2897-2902.

65. Lin P, Li J, Liu Q Mao F, Qiu R, et al. (2008) A missense mutation in SLC33A1, which encodes the acetyl-CoA transporter, causes autosomaldominant spastic paraplegia (SPG42). Am J Hum Genet 83:752-759.

66. Michel MC, Li Y, Heusch G (2001) Mitogen-activated protein kinases in the heart. Naunyn Schmiedebergs Arch Pharmacol 363:245-266.

67. Yang D, Zeng Y, Tian C, Liu J, Guo SB, et al. (2010) Transcriptomic analysis of mild hypothermia-dependent alterations during endothelial reperfusion injury. Cell Physiol Biochem 25:605-614

68. Livak KJ, Schmittgen TD (2001) Analysis of relative gene expression data using real-time quantitative PCR and the 2(-Delta Delta G(T)) Method. Methods 25:402-408 